\title{
Common Cortical and Subcortical Targets of the Dorsolateral Prefrontal and Posterior Parietal Cortices in the Rhesus Monkey: Evidence for a Distributed Neural Network Subserving Spatially Guided Behavior
}

\author{
L. D. Selemon and P. S. Goldman-Rakic \\ Section of Neuroanatomy, Yale University School of Medicine, New Haven, Connecticut 06510
}

Common efferent projections of the dorsolateral prefrontal cortex and posterior parietal cortex were examined in 3 rhesus monkeys by placing injections of tritiated amino acids and HRP in frontal and parietal cortices, respectively, of the same hemisphere. Terminal labeling originating from both frontal and parietal injection sites was found to be in apposition in 15 ipsilateral cortical areas: the supplementary motor cortex, the dorsal premotor cortex, the ventral premotor cortex, the anterior arcuate cortex (including the frontal eye fields), the orbitofrontal cortex, the anterior and posterior cingulate cortices, the frontoparietal operculum, the insular cortex, the medial parietal cortex, the superior temporal cortex, the parahippocampal gyrus, the presubiculum, the caudomedial lobule, and the medial prestriate cortex. Convergent terminal labeling was observed in the contralateral hemisphere as well, most prominently in the principal sulcal cortex, the superior arcuate cortex, and the superior temporal cortex. In certain common target areas, as for example the cingulate cortices, frontal and parietal efferents terminate in an array of interdigitating columns, an arrangement much like that observed for callosal and associational projections to the principal sulcus (Goldman-Rakic and Schwartz, 1982). In other areas, frontal and parietal terminals exhibit a laminar complementarity: in the depths of the superior temporal sulcus, prefrontal terminals are densely distributed within laminae I, III, and V, whereas parietal terminals occupy mainly laminae IV and VI directly below the prefrontal bands. Subcortical structures also receive apposing or overlapping projections from both prefrontal and parietal cortices. The dorsolateral prefrontal and posterior parietal cortices project to adjacent, longitudinal domains of the neostriatum, as has been described previously (Selemon and Goldman-Rakic, 1985); these projections are also found in close apposition in the claustrum, the amygdala, the caudomedial lobule, and throughout the anterior medial, medial dorsal, lateral dorsal, and medial pulvinar nuclei of the thalamus. In the brain stem, both areas of association cortex

\footnotetext{
Received Aug. 14, 1987; revised Mar. 7, 1988; accepted Mar. 8, 1988.

This work was supported by USPHS Grants MH38546 and MH00298 (P.S.G.-R.) and an NIA fellowship award AG05310 (L.D.S.). We wish to thank J. Coburn, M. Pappy, L. Ladewig, and J. Musco for excellent technical assistance and Dr. R. Williäms for advice on Figure 3.

Correspondence should be addressed to Dr. P. S. Goldman-Rakic, Section of Neuroanatomy, Yale University School of Medicine, 333 Cedar Street, Rm. C303 SHM, New Haven, CT 06510.

Copyright (C) 1988 Society for Neuroscience $0270-6474 / 88 / 114049-20 \$ 02.00 / 0$
}

project to the intermediate layers of the superior colliculus and to the midline reticular formation of the pons.

The present study has uncovered a remarkably large number of cortical and subcortical areas that receive input from both the dorsolateral prefrontal and posterior parietal association cortices, indicating that these common efferent pathways constitute part of an elaborate anatomical circuit which could mediate many aspects of spatial function, including spatial perception, attention, memory, and spatially guided movement.

The dorsolateral prefrontal cortex and the posterior parietal cortex are perhaps the most densely, reciprocally interconnected areas of association cortex in the primate brain (Leichnetz, 1980; Goldman-Rakic and Schwartz, 1982; Schwartz and GoldmanRakic, 1982, 1984). Moreover, a recent detailed analysis of the intracortical connections of these areas has revealed that each of the subdivisions of the posterior parietal cortex (areas $7 a, 7 b$, $7 \mathrm{ip}$, and $7 \mathrm{~m}$ ) is specifically connected with a distinct subregion of the principal sulcal cortex (Cavada and Goldman-Rakic, 1985). Thus, multiple reciprocal corticocortical projections interconnect the prefrontal and parietal cortices and provide an anatomical substrate for transfer of information between these 2 areas of higher association cortex.

The dorsolateral prefrontal cortex and the posterior parietal cortex may be related functionally as well. Physiological studies have shown that the posterior parietal cortex mediates spatial perception by transforming the retinotopic image into a map of visual space (Andersen et al., 1985) and is involved in relating body position to this map of visual space (Hyvärinen and Poranen, 1974; Mountcastle et al., 1975), while the dorsolateral prefrontal cortex plays a central role in tasks that require spatial memory, such as the delayed response and delayed alternation tasks (Goldman and Rosvold, 1970; Goldman et al., 1971; Funahashi et al., 1986). Furthermore, lesions in presumably homologous regions of the prefrontal or parietal cortex in humans result in altered spatial perception and often in sensory neglect of space contralateral to the lesion (Holmes, 1918; Denny-Brown and Banker, 1952; Critchley, 1953; Hécaen et al., 1956; Heilman and Valenstein, 1972).

In the present study we have examined the common outflow pathways of the dorsolateral prefrontal and posterior parietal cortices using a double anterograde labeling method that allowed us to visualize the prefrontal and parietal projections in adjacent sections. While numerous studies have traced the efferent connections of each of these cortical areas (see Table 1), the present 


\begin{tabular}{|c|c|c|}
\hline Target areas & Prefrontal & Parietal \\
\hline $\begin{array}{l}\text { Supplementary motor area } \\
\text { (area 6) }\end{array}$ & $\begin{array}{l}\text { Pandya and Kuypers, } 1969^{a} \\
\text { Pandya et al., 1971 } \\
\text { Jürgens, } 1984^{b}\end{array}$ & $\begin{array}{l}\text { Pandya and Kuypers, 1969a } \\
\text { Petrides and Pandya, 1984 } \\
\text { Jürgens, } 1984^{b}\end{array}$ \\
\hline $\begin{array}{l}\text { Dorsal premotor cortex } \\
\quad(\text { area } 6 \alpha)\end{array}$ & $\begin{array}{l}\text { Pandya and Kuypers, } 1969 \\
\text { Pandya et al., } 1971 \\
\text { Barbas and Pandya, } 1987^{\circ}\end{array}$ & $\begin{array}{l}\text { Pandya and Kuypers, } 1969 \\
\text { Chavis and Pandya, } 1976 \\
\text { Petrides and Pandya, } 1984\end{array}$ \\
\hline $\begin{array}{l}\text { Ventral premotor cortex } \\
\quad(\text { area } 6 \beta)\end{array}$ & $\begin{array}{l}\text { Pandya and Kuypers, } 1969 \\
\text { Pandya et al., } 1971 \\
\text { Matelli et al., 1986 } \\
\text { Barbas and Pandya, } 1987^{\circ}\end{array}$ & $\begin{array}{l}\text { Pandya and Kuypers, } 1969 \\
\text { Chavis and Pandya, } 1976 \\
\text { Petrides and Pandya, } 1984 \\
\text { Godschalk et al., } 1984^{b} \\
\text { Matelli et al., } 1986^{b}\end{array}$ \\
\hline $\begin{array}{l}\text { Anterior arcuate cortex } \\
\quad \text { (area 8) }\end{array}$ & $\begin{array}{l}\text { Pandya and Kuypers, } 1969 \\
\text { Pandya et al., } 1971 \\
\text { Goldman and Nauta, 1977a } \\
\text { Barbas and Mesulam, } 1981^{b} \\
\text { Huerta et al., } 1987^{\circ}\end{array}$ & $\begin{array}{l}\text { Pandya and Kuypers, } 1969 \\
\text { Jones and Powell, } 1970 \\
\text { Chavis and Pandya, } 1976 \\
\text { Barbas and Mesulam, } 1981^{b} \\
\text { Petrides and Pandya, } 1984 \\
\text { Huerta et al., } 1987^{\circ} \\
\end{array}$ \\
\hline $\begin{array}{l}\text { Orbitofrontal cortex } \\
\text { (area 11) }\end{array}$ & $\begin{array}{l}\text { Pandya et al., } 1971 \\
\text { Goldman and Nauta, 1977a }\end{array}$ & Cavada and Goldman-Rakic, 1986 \\
\hline $\begin{array}{l}\text { Anterior cingulate cortex } \\
\text { (area 24) }\end{array}$ & $\begin{array}{l}\text { Pandya and Kuypers, } 1969 \\
\text { Pandya et al., } 1971 \\
\text { Goldman and Nauta, } 1977 \mathrm{a} \\
\text { Baleydier and Mauguiere, } 1980^{b}\end{array}$ & $\begin{array}{l}\text { Pandya and Kuypers, } 1969 \\
\text { Jones and Powell, } 1970 \\
\text { Baleydier and Mauguiere, } 1980^{\circ} \\
\text { Petrides and Pandya, } 1984\end{array}$ \\
\hline $\begin{array}{l}\text { Posterior cingulate cortex } \\
\quad(\text { area 23) }\end{array}$ & $\begin{array}{l}\text { Pandya and Kuypers, } 1969 \\
\text { Pandya et al., } 1971 \\
\text { Goldman and Nauta, 1977a } \\
\text { Baleydier and Mauguiere, } 1980^{b}\end{array}$ & $\begin{array}{l}\text { Pandya and Kuypers, } 1969 \\
\text { Jones and Powell, } 1970 \\
\text { Baleydier and Mauguiere, } 1980^{b} \\
\text { Petrides and Pandya, } 1984\end{array}$ \\
\hline $\begin{array}{l}\text { Frontoparietal operculum } \\
\quad \text { (areas } 1,2, \mathrm{SII})\end{array}$ & $\begin{array}{l}\text { Pandya et al., } 1971 \\
\text { Preuss and Goldman-Rakic, } \\
1985\end{array}$ & $\begin{array}{l}\text { Pandya and Kuypers, } 1969 \\
\text { Pandya and Seltzer, } 1982 \\
\text { Petrides and Pandya, } 1984\end{array}$ \\
\hline Insular cortex (area IG) & $\begin{array}{l}\text { Pandya et al., } 1971 \\
\text { Goldman and Nauta, 1977a } \\
\text { Mufson and Mesulam, } 1982^{b}\end{array}$ & Mufson and Mesulam, $1982^{b}$ \\
\hline $\begin{array}{l}\text { Medial parietal cortex } \\
\text { (area } 7 \mathrm{~m} \text { ) }\end{array}$ & Goldman and Nauta, 1977 $\mathbf{a}^{c}$ & $\begin{array}{l}\text { Pandya and Kuypers, } 1969^{a} \\
\text { Pandya and Seltzer, } 1982\end{array}$ \\
\hline $\begin{array}{l}\text { Superior temporal cortex } \\
\quad \text { (area IPa) }\end{array}$ & $\begin{array}{l}\text { Pandya and Kuypers, } 1969 \\
\text { Pandya et al., } 1971 \\
\text { Goldman and Nauta, 1977a }\end{array}$ & $\begin{array}{l}\text { Pandya and Kuypers, } 1969 \\
\text { Jones and Powell, } 1970 \\
\text { Seltzer and Pandya, } 1978 \\
\text { Seltzer and Pandya, } 1984\end{array}$ \\
\hline Presubiculum & Goldman-Rakic et al., 1984 & $\begin{array}{l}\text { Seltzer and Van Hoesen, } 1979 \\
\text { Seltzer and Pandya, } 1984\end{array}$ \\
\hline $\begin{array}{l}\text { Parahippocampal gyrus } \\
\text { (area TF) }\end{array}$ & $\begin{array}{l}\text { Pandya and Kuypers, } 1969 \\
\text { Pandya et al., } 1971 \\
\text { Goldman-Rakic et al., } 1984\end{array}$ & $\begin{array}{l}\text { Jones and Powell, } 1970 \\
\text { Seltzer and Pandya, } 1984\end{array}$ \\
\hline Caudomedial lobule & Goldman-Rakic et al., 1984 & Cavada and Goldman-Rakic, 1986 \\
\hline $\begin{array}{l}\text { Medial prestriate cortex } \\
\text { (area 19) }\end{array}$ & NPD & Cavada and Goldman-Rakic, 1986 \\
\hline Neostriatum & $\begin{array}{l}\text { Goldman and Nauta, 1977b } \\
\text { Selemon and Goldman-Rakic, } \\
1985\end{array}$ & Yeterian and Van Hoesen, 1978 \\
\hline Amygdala & Aggleton et al., $1980^{b}$ & Aggleton et al., $1980^{b}$ \\
\hline Claustrum & Pearson et al., 1982 & Pearson et al., 1982 \\
\hline
\end{tabular}




\begin{tabular}{|c|c|c|}
\hline Target areas & Prefrontal & Parietal \\
\hline Thalamus & $\begin{array}{l}\text { Jacobsen et al., } 1978 \\
\text { Künzle, } 1978 \\
\text { Preuss and Goldman-Kakic, } \\
1987\end{array}$ & $\begin{array}{l}\text { Asanuma et al., } 1985 \\
\text { Yeterian and Pandya, } 1985\end{array}$ \\
\hline Superior colliculus & $\begin{array}{l}\text { Kuypers and Lawrence, } 1967 \\
\text { Goldman and Nauta, } 1976 \\
\text { Leichnetz et al., } 1981^{b}\end{array}$ & $\begin{array}{l}\text { Kuypers and Lawrence, } 1967 \\
\text { Asanuma et al., } 1985 \\
\text { Lynch et al., } 1985\end{array}$ \\
\hline Pontine reticular formation & Brodal, 1980 & $\begin{array}{l}\text { Kuypers and Lawrence, } 1967 \\
\text { Leichnetz et al., } 1984^{\circ}\end{array}$ \\
\hline
\end{tabular}

NPD, not previously described.

a Projections that were shown in published diagrams but not included in the descriptive passage of the text.

${ }^{b}$ Evidence for projections based on placement of retrograde tracer in the target area.

c Target area referred to as retrosplenial cortex.

study has focused more specifically on the efferent target areas that receive projections from both prefrontal and parietal cortices. A double-labeling paradigm enabled us to identify the areas in which integration of prefrontal and parietal projections may occur and to examine the interrelationship of the dorsolateral prefrontal and posterior parietal terminal fields in these common target areas.

\section{Materials and Methods}

Injections. A double anterograde labeling paradigm was used to examine the common efferent targets of the dorsolateral prefrontal cortex (Brodmann's area 9,10) and the posterior parietal cortex (Brodmann's area 7) in 3 rhesus monkeys. All injections were made in the left hemisphere. In case 1 tritiated amino acids $\left({ }^{3} \mathrm{H}-\mathrm{AA}\right)$ were injected into the dorsal bank of the principal sulcus, and HRP pellets were placed in the posterior bank of the intraparietal sulcus during the same surgery. In case $2,{ }^{3} \mathrm{H}$ AA were injected into both dorsal and ventral banks of the principal sulcus; $10 \mathrm{~d}$ later multiple small injections of wheat germ agglutininhorseradish peroxidase (WGA-HRP) were made in the posterior parietal cortex. Placement of tracers was reversed in case 3: ${ }^{3} \mathrm{H}$-AA were injected in the posterior parietal cortex and HRP pellets were implanted 1 week later in the dorsolateral prefrontal cortex. The reversal of tracers in this case served as a control for the spread of tracer into the white matter underlying the parietal cortex in the previous 2 cases. Since tritiated amino acids are not taken up by cut axons, we could eliminate from consideration any parietal projections that were observed only with HRP tracers. As it turned out, however, the 2 methods produced the same findings for comparable prefrontal and parietal injections, and the results obtained with both techniques were entirely consistent with previous reports based on smaller injection sites in the prefrontal or parietal cortex (see Table 1). Monkeys were sacrificed $2 \mathrm{~d}$ following HRP injection or implants; the interval between injection of ${ }^{3} \mathrm{H}-\mathrm{AA}$ and sacrifice ranged from $2-12 \mathrm{~d}$.

Perfusion. Following deep barbituate anesthesia, monkeys were perfused with 1-2 liters of PBS followed by a mixed aldehyde fixative in phosphate buffer $(1.0 \%$ paraformaldehyde $+2.5 \%$ glutaraldehyde for cases 1 and 3; the concentration of glutaraldehyde was reduced to $1.25 \%$ in case 2). Postfixation rinses consisted of a scrics of graded sucrose solutions in phosphate buffer. The $\mathrm{pH}$ of all perfusate solutions was 7.4. Saline rinses and fixatives were warmed to $37^{\circ} \mathrm{C}$; postfixation sucrose rinses were cooled to $4^{\circ} \mathrm{C}$.

Histology. The brains were sectioned at 40 or $50 \mu \mathrm{m}$ on a freezingstage microtome, and adjacent sections were processed for autoradiographic localization of ${ }^{3} \mathrm{H}-\mathrm{AA}$ and HRP histochemistry, respectively. In cases 1 and 3 autoradiograms were counterstained with thionin. In case 2 a separate set of sections, adjacent to those processed for autoradiographic localization of ${ }^{3} \mathrm{H}$-AA, were Nissl-stained with cresyl violet.

Autoradiography. Equal amounts of tritiated leucine (specific activity $>110 \mathrm{Ci} / \mathrm{mmol}$ ) and proline (specific activity $20-40 \mathrm{Ci} / \mathrm{mmol}$ ) were evaporated and then reconstituted in distilled water to a final concen- tration of $100 \mu \mathrm{Ci} / \mu \mathrm{l}$. Multiple small injections of radiolabeled isotope were made in the desired cortical area at depths of 3.0 and $1.5 \mathrm{~mm}$ from the cortical surface. The total amount of isotope injected in each monkey equaled 360,390 , and $450 \mu \mathrm{Ci}$, respectively. Frozen sections were mounted on glass slides and coated with tritium-sensitive emulsion, then exposed for $12-16$ weeks at $-20^{\circ} \mathrm{C}$ in the dark, and developed as described in detail by Goldman and Nauta (1977a).

$H R P$ histochemistry. HRP pellets were prepared according to the method of Griffin et al. (1979). In cases 1 and 3, 7-8 pellets were placed in the selected cortical site just below the pial surface. In case 2,5 injections totaling $1.6 \mu \mathrm{l}$ in a volume of a $1.5 \%$ solution of WGA-HRP were placed $1.0 \mathrm{~mm}$ below the cortical surface. Sections were reacted for HRP with the tetramethylbenzidine method of Mesulam (1978).

Data analysis. Adjacent autoradiograms and HRP-reacted sections were examined, and selected pairs were photographed, under low power, dark-field illumination on a Leitz Orthoplan microscope; Nissl-stained sections were viewed under bright-field illumination. Localization of terminal labeling to a specific cortical area or subcortical nucleus was based on cytoarchitectonic analysis of each area in conjunction with an evaluation of the sulcal patterning of the section. Autoradingraphic silver grains and HRP labeling in adjacent sections were drawn with the aid of a Wild zoom stereomicroscope equipped with a camera lucida attachment. Use of the zoom stereomicroscope permitted us to vary the magnification of the HRP sections to match the adjacent autoradiograms in order to compensate for differences in size due to differential processing. These drawings then were superimposed to create composite drawings of the parietal and prefrontal labeling at each level.

The flattened reconstruction of prefrontal and parietal labeling of the arcuate premotor cortex in Figure 3 is based on drawings of adjacent sections through this region from case 2 . For each pair of sections, the outer cortical surface was traced on the graphics tablet of a MacIntosh computer. The fundus of the principal sulcus arbitrarily was designated as zero, or in sections posterior to the sulcus, the midpoint between the ventral lip of the superior arcuate sulcus and the dorsal lip of the inferior arcuate sulcus was assigned the zero value. The distance between the zero point and the fundus of the superior arcuate sulcus, as well as the approximate location of the dorsal and ventral rims, was measured and assigned a positive value. Distances between zero and analogous landmarks of the inferior arcuate sulcus were assigned negative values. Subsequently, the location of parietal and prefrontal terminal labeling in layer I was determined by measuring the distance between the label and the nearest fundus, i.e., of the principal sulcus, superior arcuate sulcus, or inferior arcuate sulcus. All measurements were then graphed to show the distribution of prefrontal and parietal label in the arcuate cortex. Reconstructions were made of layer I, rather than of layer IV, because terminal labeling was more consistently found in layer I and because much of the cortex in this region was cut tangentially in frontal section, making the identification of deeper layers difficult. While we recognize that basing the reconstructions on layer I introduces distortion into the map such that the cortex deep within each sulcus is compressed and the cortex on the rims and convexities is expanded, we feel that the reconstructions accurately depict the interrelationship of prefrontal and parietal terminal labeling in the arcuate cortex. 


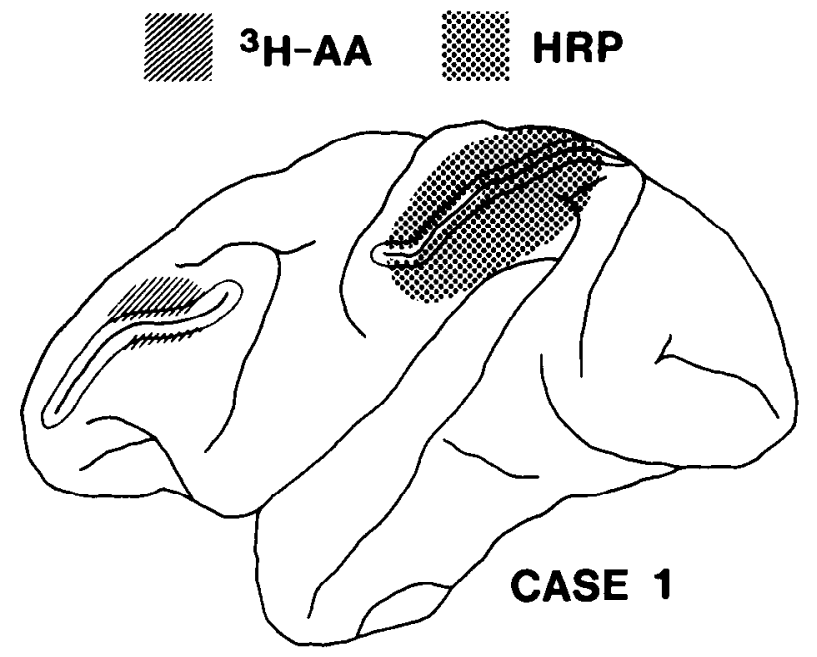

into the adjacent dorsal and ventral cortex and rostrally to include the ventral lip of the sulcus. In case 3, the HRP injection site covered much of the dorsolateral frontal cortex with the cxclusion of the banks and depths of the rostral third of the principal sulcus. The halo of the injection site involved the ventral bank of the superior arcuate sulcus (area 6) and the cortex posterior to the principal sulcus (area 8); in addition, there was spread of HRP through the white matter to involve the cortex surrounding the lateral orbital sulcus (area 11) and the rostral cingulate sulcus (areas 6, 32) (not shown in Fig. 1). In all cases, the parietal injection sites were centered in the lateral parietal cortex bordering the posterior bank of the intraparietal sulcus (area 7). However, the 3 injection sites differed with respect to the relative involvement of the various subdivisions of Brodmann's area 7, designated 7a, 7b, 7ip by Cavada and GoldmanRakic (1985). In case 1, the injection site included the cortex dorsal and ventral to the intraparietal sulcus along nearly its entire length (areas 5, 7a, b); HRP reaction product filled the banks and fundus of the posterior half of the sulcus as well (area 7ip). The HRP injection site in case 2 was more limited involving only the cortex bordering the posterior two-thirds of the sulcus (areas 7a, caudal 7b). In case 3, silver grains were concentrated in the posterior parietal cortex bordering the middle of the intraparietal sulcus (areas $7 a, b$ ), but there was substantial involvement of the posterior bank of the sulcus (area 7ip) and spread of tritiated amino acids into the cortex dorsal to the sulcus at rostral levels (area 5).

\section{Technical considerations}

The following description of common efferent targets is based largely on the most optimal case (case 2) in which the frontal injection site was restricted to areas 9 and 10 and the parietal inejction site to area 7 . Only about half of these projections were observed in case 1. In part, this was due to technical limitations: for example, HRP-reacted sections were not collected rostral to the anterior limit of the arcuate sulcus because the case was originally processed to examine common corticostriatal projections; likewise, a portion of the rostromedial temporal lobe was excised for another study. However, the absence of frontal projections in and around the inferior arcuate sulcus and to the frontoparietal operculum and superior temporal cortex in case 1 may indicate that these projections arise either from the depths of the principal sulcus or from the cortex ventral to the sulcus. Although the frontal injection site in case 3 was not restricted to areas 9 and 10 , very few additional common projections were observed, notably to the superior colliculus and the caudomedial lobule. Since these projections were present in case 1 as well, it is possible that they originate from the cortex dorsal to the principal sulcus and from the posterior bank of the intraparietal sulcus (area 7ip), areas that were not injected in case 2.

Some mention of the nonconvergent projections of the dorsolateral prefrontal and posterior parietal cortices should be made, although these were not analyzed in detail in the present study. In general, frontal projections to other frontal areas were more extensive than comparable parietal projections. For example, in cases 2 and 3 (no data are available for case 1), projections from the prefrontal cortex to the cingulate and dorsomedial premotor cortices extended much farther rostrally than those originating in the posterior parietal cortex. Conversely, in all cases parietal projections to the temporal and occipital cortices were more widespread than projections from the frontal cortex. Parietal projections extended onto the dorsal and ventral banks of the superior temporal sulcus, although the common 

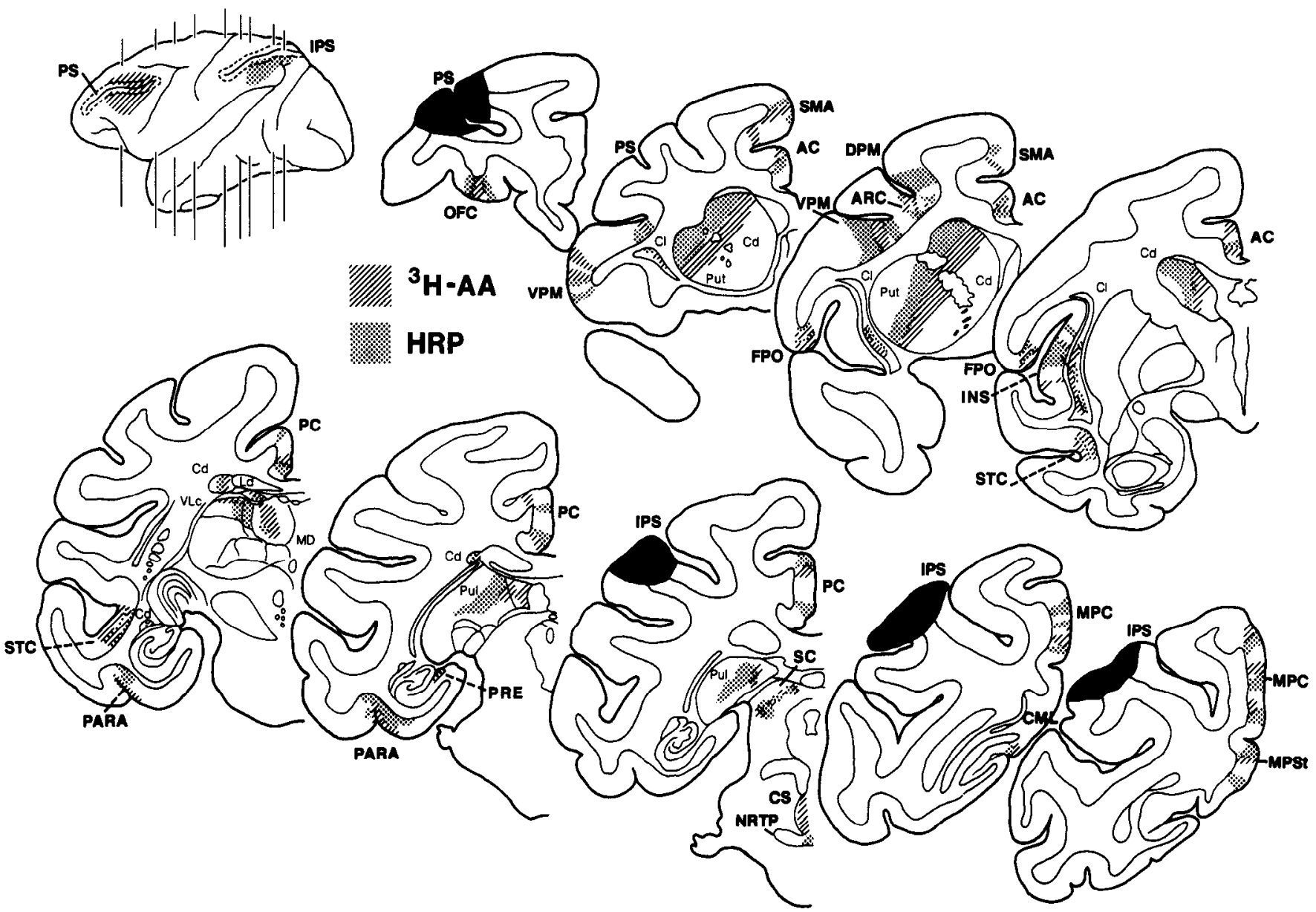

Figure 2. Common target areas of the prefrontal and parietal cortices shown in 9 coronal sections through the rhesus monkey brain. All of the projections depicted were observed in case 2 (injection sites shown on a lateral view of the brain) with the exception of the projections to the caudomedial lobule, the medial prestriate cortex, the superior colliculus, and the pontine reticular formation, which were observed in case 3 . Nonconvergent projections are not shown on this semischematic diagram. Autoradiographic label representing prefrontal terminal labeling is indicated by cross-hatching, HRP reaction product representing parietal terminal labeling is illustrated by stippling. Abbreviations: $A C$, anterior cingulate cortex; $A R C$, arcuate cortex; $C d$, caudate nucleus; $C l$, claustrum; $C M L$, caudomedial lobule; $C S$, central superior nucleus; $D P M$, dorsal premotor cortex; $F P O$, frontoparietal operculum; INS, insular cortex; IPS, intraparietal sulcus; $L D$, lateral dorsal nucleus of the thalamus; $M D$, medial dorsal nucleus of the thalamus; $M P C$, medial parietal cortex; MPSt, medial prestriate cortex; NRTP, nucleus reticularis tegmenti pontis; $O F C$, orbitofrontal cortex; PARA, parahippocampal cortex; $P C$, posterior cingulate cortex; $P R E$, presubiculum; $P S$, principal sulcus; $P u l$, pulvinar nucleus of the thalamus; Put, putamen; $S C$, superior colliculus; $S M A$, supplementary motor area; $S T C$, superior temporal cortex; VPM, ventral premotor cortex; $V L c$, ventral lateral nucleus of the thalamus, pars caudalis.

efferent target of the 2 cortices was restricted to the depths of the sulcus. Parietal cortex also projected to the occipital cortex surrounding the lunate and occipitotemporal sulci, areas that were not innervated by frontal terminals in these cases. In this regard, projections to the contralateral hemisphere also warrant consideration. The dorsolateral prefrontal cortex projected preferentially to homotopic and many heterotopic areas of the frontal cortex, and widespread projections throughout the contralateral hemisphere were observed from the posterior parietal cortex. On the whole, these contralateral projections were nonconvergent. However, a few areas of convergence were observed in the opposite hemisphere: Dual terminal labeling from the prefrontal and parietal injection sites was observed in the contralateral principal sulcal and arcuate prefrontal cortices and in the depths of the superior temporal sulcus.

As the injection sites in this study were made intentionally large to expose the full extent of the efferent circuitry of the dorsolateral prefrontal and posterior parietal cortices, some of them impinged on adjacent areas of cortex, and in all cases, spread into the underlying white matter to varying degrees.
However, comparison of the patterns of projection among the 3 cases enabled us to identify and dismiss those projections that did not originate in the intended injection areas. Thus, in case 1 with extensive involvement of area 5, terminal labeling from the parietal injection site was present in the primary somatosensory and motor cortices but was not observed in case 2 , whose injection was confined to area 7. Furthermore, as known from the literature, the dorsolateral prefrontal cortex does not project to either the primary motor or somatosensory cortex and hence convergence with parietal terminals was neither expected nor observed in these 2 areas.

\section{Projections}

\section{Cortical targets}

Fifteen cortical areas received projections from both prefrontal and parietal injection sites in the ipsilateral hemisphere (Fig. 2). Brodmann's areal designations are used in the following description except where otherwise indicated. In the frontal lobe, common targets included the supplementary motor cortex (area 6 ), the dorsal premotor cortex (area $6 \alpha$ of Vogt), the ventral 

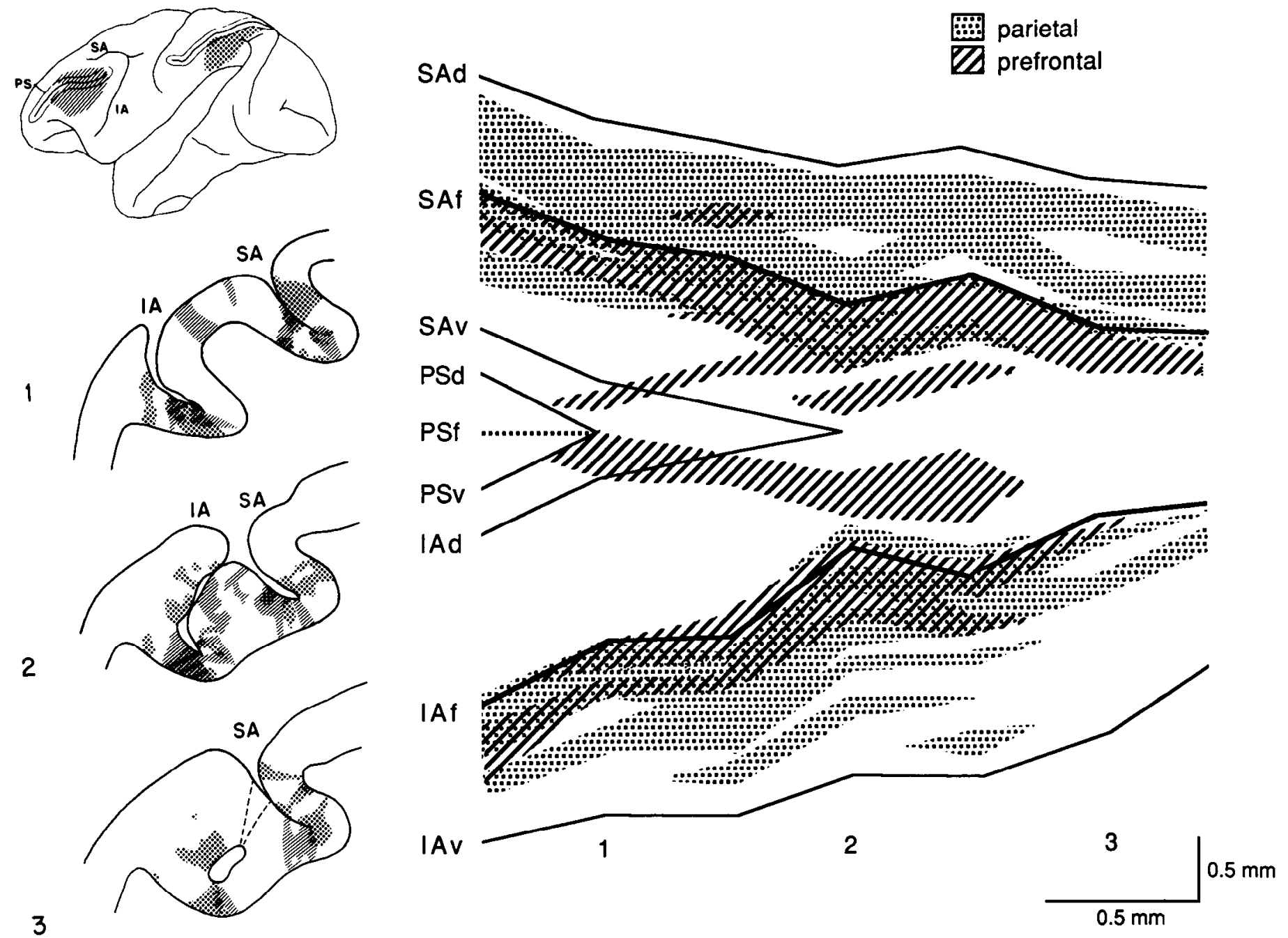

Figure 3. Composite 2-dimensional reconstruction of prefrontal and parietal terminal fields in layer I of the arcuate cortex, including the frontal eye fields, from case 2. The prefrontal and parietal injection sites are shown on a lateral brain view above, and drawings of labeling in 3 frontal sections are illustrated below. Cross-hatching represents the autoradiographic label from the frontal injection site; stippling indicates HRP terminal labeling from the parietal projection. Note that the prefrontal and parietal projections form parallel sets of stripes across the cortical surface with some overlap at borders between adjacent stripes. The overlap is more prominent in layer I than in other layers. Abbreviations: $I A$, inferior arcuate sulcus; $P S$, principal sulcus; $S A$, superior arcuate sulcus; $d l$, dulcus; $v l$, ventral; $f$, fundus.

premotor cortex (area $6 \beta$ of Vogt), the anterior bank of the arcuate sulcus (area 8), the anterior cingulate cortex (area 24), and the orbital prefrontal cortex (area 11 ). In area 8 , dual terminal labeling was observed throughout the anterior banks and depths of the superior and inferior arcuate sulci and in the intervening cortex, including the frontal eye fields (Figs. 2, 3). In addition, labeling was observed in the dorsal premotor cortex in the posterior banks of the arcuate sulci (Figs. 2, 3). Prefrontal and parietal terminal labeling also extended into the ventral premotor cortex in the posterior hank of the inferior arcuate sulcus and onto the convexity of the hemisphere just ventral to this sulcus (Figs. 2, 3). On the medial surface of the hemisphere, the 2 projection fields were closely apposed in a limited portion of the premotor cortex roughly corresponding to the area that has been physiologically defined as the supplementary motor area (Figs. 2; 4, $A, B$ ). Ventral to the cingulate sulcus, prefrontal and parietal terminal fields were interrelated in the caudal half of the anterior cingulate gyrus (Figs. 2; 4, C, D). In the orbitofrontal cortex, the common projection area was confined to the superficial cortex between the medial and lateral orbital sulci (Fig. 2).
At the juncture between frontal and parietal lobes, prominent terminal labeling from both injection sites was present in the upper bank of the Sylvian fissure (the frontoparietal operculum) and in the insular cortex proper (area IG of Jones and Burton, 1976). In the frontoparietal operculum, prefrontal and parietal terminal labeling extended from nearly its anterior limit to well beyond the level of its intersection with the central sulcus. [Note that this projection probably encompassed more than one cytoarchitectonic area, i.e., SI (Brodmann's areas 1,2) and SII.] The common target area within the operculum was located approximately midway between the dorsal lip of the Sylvian fissure and the insular cortex (Figs. 2; 5, $A, B$ ). Labeling was less extensive in the insular cortex and located near the level of the central sulcus (Figs. 2; 9, C,D). On the medial surface of the parieto-occipital lobe, the common efferent targets of the prefrontal and parietal projections included the entire posterior cingulate gyrus (area 23) and the medial parietal cortex (area 7m) (Figs. 2, 6).

In the temporal lobe, dual terminal labeling was most prominent in the depths of the superior temporal sulcus (area IPa of Seltzer and Pandya, 1978) where it was confined to the middle 

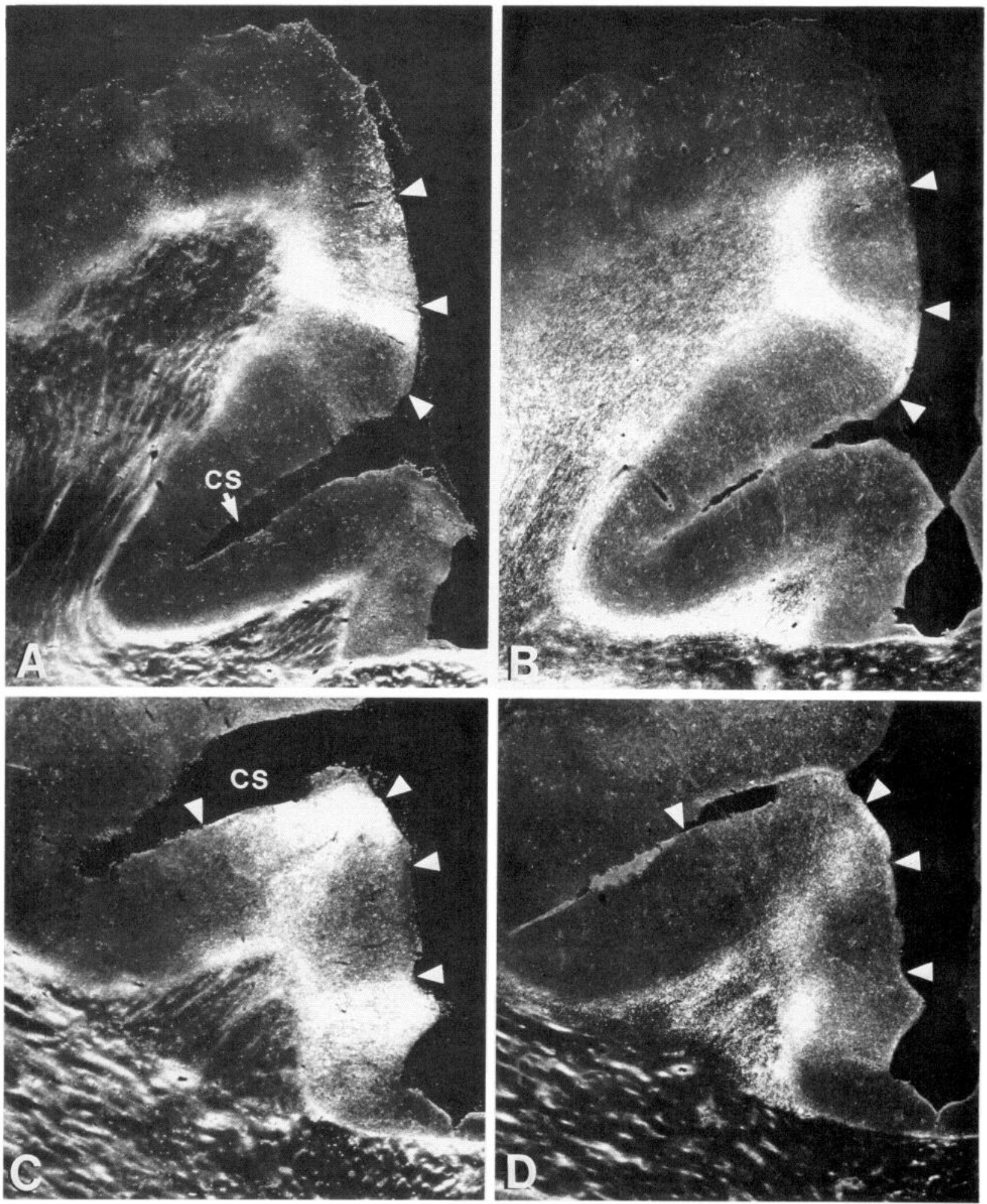

Figure 4. Paired dark-field photomicrographs of adjacent sections through the dorsomedial and anterior cingulate cortices in case 2. $A$, In the dorsomedial cortex, parietal terminals form a dense, broad column on the medial surface of the hemisphere. $B$, In an adjacent section, a dense column of prefrontal terminal labeling is situated ventral to the parietal column, and a smaller, weaker prefrontal column is present dorsally. $C$, In the anterior cingulate cortex, broad columns of parietal terminals flank $(D)$ a smaller prefrontal column. Arrowheads point to surface landmarks that aid in comparison of adjacent sections. Abbreviation: $C S$, cingulate sulcus. Magnification: $A$ and $B, \times 9.5 ; C$ and $D, \times 12.8$.

third of the sulcus (Figs. 2; 5, C, D). Caudally within the temporal lobe, prefrontal and parietal projections were observed in the presubiculum (Figs. 2, 7A) and in the parahippocampal cortex, area TF of von Bonin and Bailey (1947) (Fig. 2). In cases
1 and 3, prefrontal and parietal terminals were present in a small outpouching of cortex adjacent to the posterior hippocampus designated the caudomedial lobule (Goldman-Rakic et al., 1984) (Figs. 2, 7B). Finally, a smaller common projection 

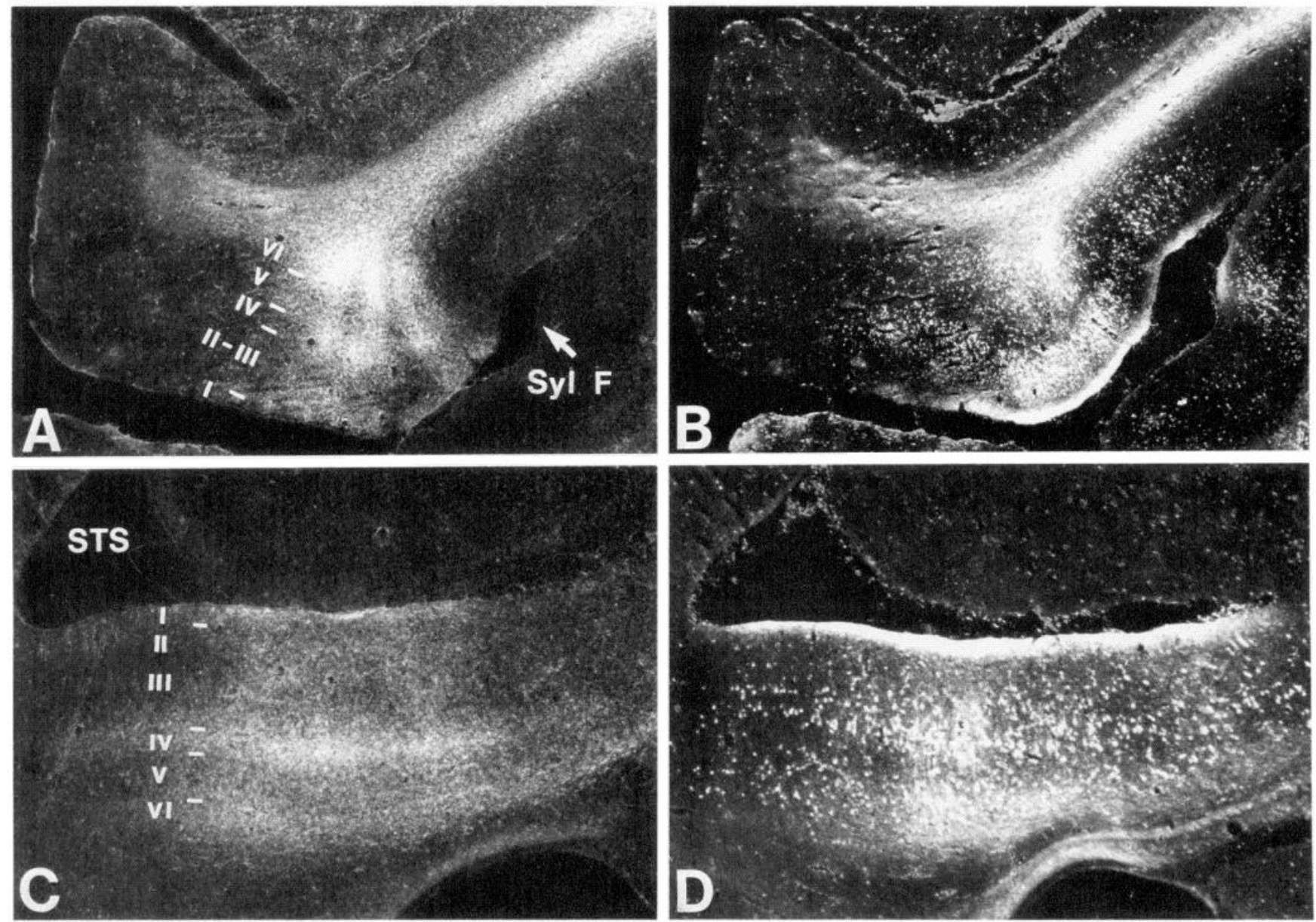

Figure 5. Dark-field photomicrographs of the frontoparietal operculum and the superior temporal sulcus in case 3 . $A$, In the frontoparietal operculum, parietal terminals are most densely distributed in layer IV, where 3 dense patches of label are found, and in layer VI. $B$, In an adjacent section, prefrontal terminals form a dense band in layer I with patches of terminal labeling located directly below in layers III and V. $C$, Parietal terminal labeling is also concentrated in layers IV and VI in the depths of the superior temporal sulcus; $D$, prefrontal terminal labeling is most extensive in layers I, V, and III, respectively. Note that a subtle columnar patterning is superimposed on the more prominent laminar segregation as dense clusters of prefrontal labeling in layers III and V are aligned in a vertical column that is flanked by dense clusters of parietal labeling in layers IV and VI. Abbreviations: $S T S$, superior temporal sulcus; $S y l F$, Sylvian fissure. Magnification: $A$ and $B, \times 9.6 ; C$ and $D, \times 8$.

area was observed posterior to the caudomedial lobule in the medial prestriate cortex of the occipital lobe (area 19) in case 3 (Fig. 2),

Parietal and prefrontal projections converged to a more limited extent in the contralateral hemisphere. For example, in case 2 parietal terminal labeling was observed in the ventral bank and rim of the contralateral principal sulcus, which also received a dense homotopic projection from the prefrontal injection site in this case. Farther posterior in the frontal lobe in case 2, columns of prefrontal and parietal terminal labeling were apposed in the anterior bank of the superior arcuate sulcus. Beyond the central sulcus, a small prefrontal projection to the cortex in the depths of the contralateral superior temporal sulcus, an area which receives bilateral innervation from the posterior parietal cortex, was seen in case 3 . Although dual terminal labeling was not observed in the insular cortex in any case, a frontal projection to the insular cortex was present in case 3 , and a parietal projection was noted in case 2 . It is therefore possible that the insular cortex, and perhaps other areas of the contralateral hemisphere, might be added to the list of common efferent targets if more sections were collected and analyzed.
We cannot exclude the possibility that a few additional areas of the ipsilateral hemisphere may receive prefrontal and parietal input, as well. One area that is known to receive input from both cortices is the entorhinal cortex in the depth of the rhinal sulcus (Jones and Powell, 1970; Goldman-Rakic et al., 1984; Seltzer and Pandya, 1984). Although technical difficulties and considerations limited our evaluation of the rostromedial temporal lobe, prefrontal terminal labeling was observed in the rhinal sulcus of case 3 , and parietal terminal labeling was found in this same area in case 1. It seems unlikely that any other common target areas in the ipsilateral hemisphere would have been missed in the present study since all other areas of the brain were analyzed in detail in at least 2 of the 3 cases. Moreover, the projections described in the present study are in agreement with the results of previous single-labeling studies (see Table 1).

Columnar interdigitation of prefrontal and parietal terminal fields. In most cortical target areas, columns of terminal labeling originating from the frontal injection site alternated spatially with similar columns of parietal labeling. Although overlap of prefrontal and parietal terminal labeling was observed, inter- 

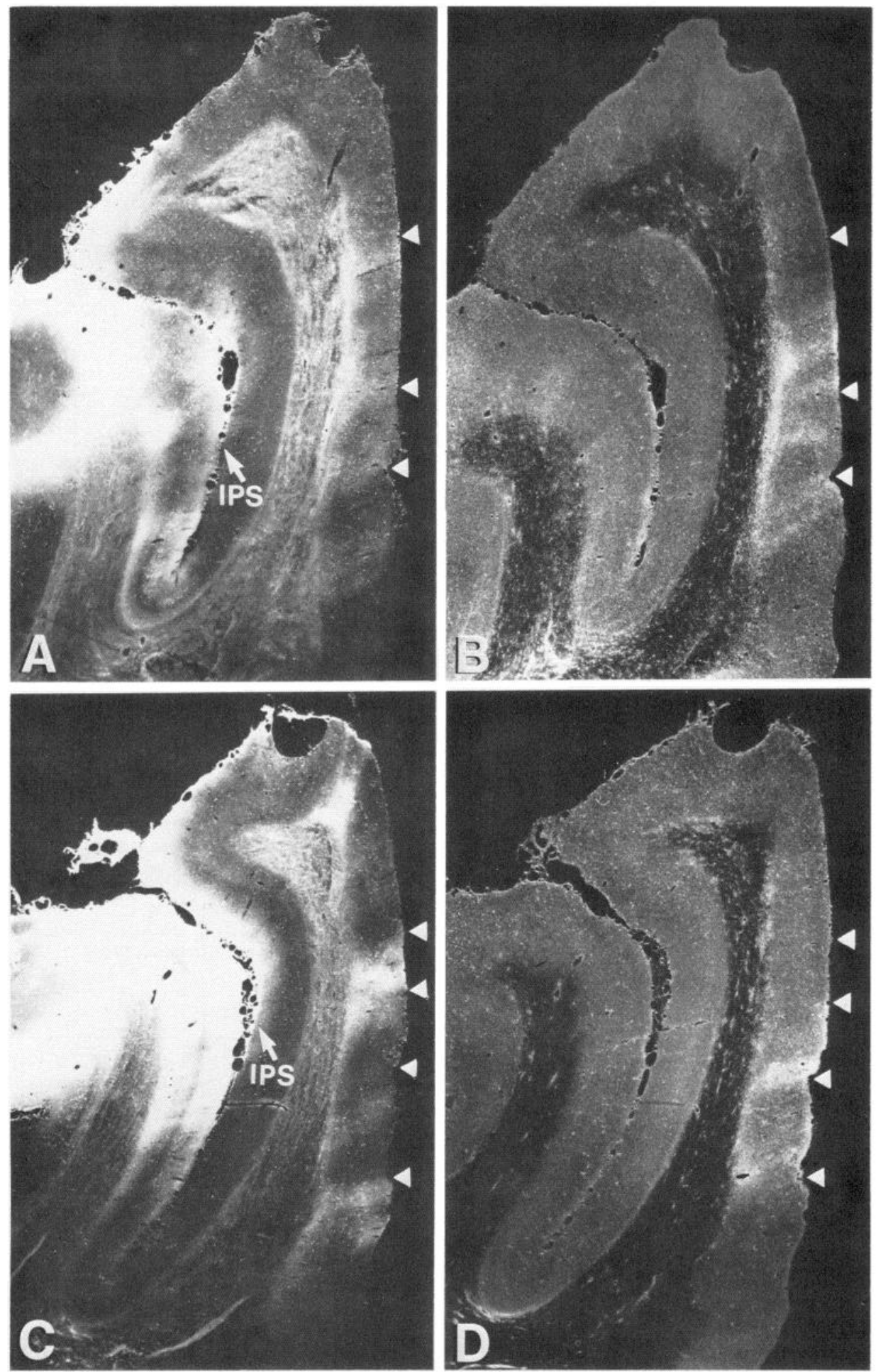

Figure 6. Dark-field photomicrographs showing interdigitation of prefrontal and parietal columns in area $7 \mathrm{~m}$ in case 2. A, Three faint columns of parietal terminals are located along the medial surface of the hemisphere. $B$, In an adjacent section, multiple columns of prefrontal terminal labeling are present, and these appear to be interposed between the parietal columns. In $C$, three widely spaced columns of parietal terminals are shown in area $7 \mathrm{~m}$, while in $D$, a pair of prefrontal columns are located between 2 of the parietal columns in an adjacent section. Arrowheads serve as points of reference for comparison of the 2 sections. Note that a portion of the parietal injection site is visible in $A$ and $C$. IPS, intraparietal sulcus. $\times 6.8$.

digitation of prefrontal and parietal columns appeared to be the preponderant arrangement of these projections. Very little laminar specificity was discernible in the columnar projections with the exception that layer I often was more densely labeled than other layers in both prefrontal and parietal columns. Columnar patterning was particularly prominent in the anterior (Figs. 2; $4, C, D$ ) and posterior cingulate gyrus (Fig. 2) where interdigi- tation of prefrontal and parietal columns extended from the middle of area 24 throughout the entire extent of area 23 to well beyond the splenium of the corpus callosum and therefore including the medial portion of area 7 (Figs. 2, 6). In many instances, adjacent prefrontal and parietal columns traversed several sections, which were $400 \mu \mathrm{m}$ apart. This same columnar relationship was observed in a limited portion of the supple- 

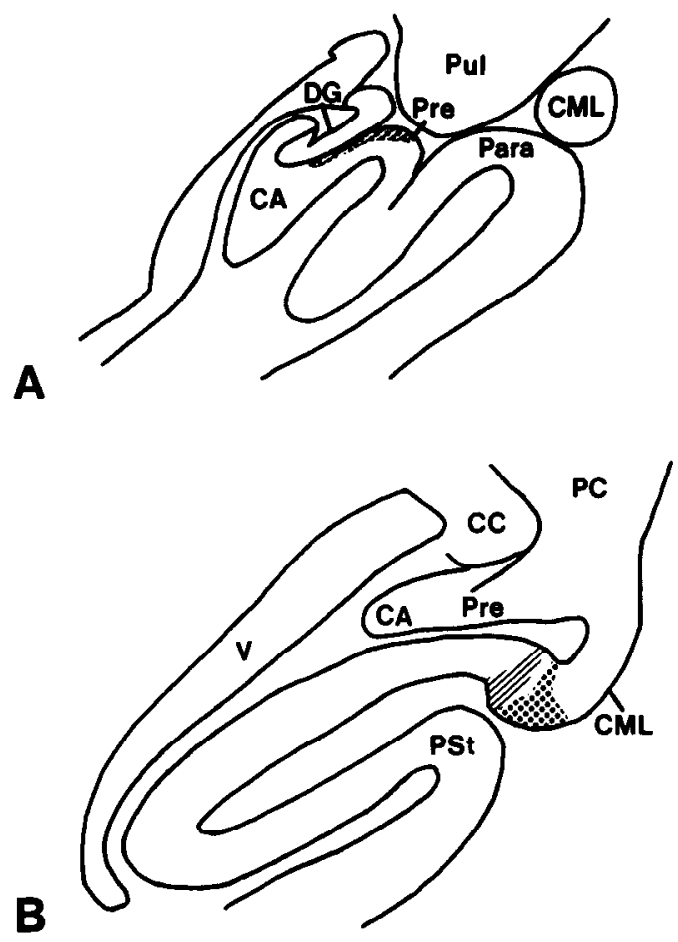

Figure 7. Drawing of prefrontal and parietal projection to the temporal lobe in case 3. $A$, In the presubiculum, prefrontal and parietal terminals overlap in the molecular layer at posterior levels of the hippocampal gyrus. $B$, A column of prefrontal terminals (stippling) is adjacent to a columnar projection from the parietal cortex (cross-hatching) in the caudomedial lobule. Abbreviations: $C A$, Ammons horn; $C C$, corpus callosum: $C M L$, caudomedial lobule; $D G$, dentate gyrus; Para, parahippocampal cortex; $P C$, posterior cingulate cortex; Pre, presubiculum; $P S t$, prestriate cortex; Pul, pulvinar.

mentary motor cortex on the medial surface of the hemisphere (Figs. $2 ; 4, A, B$ ). In the cortex posterior to the principal sulcus, i.c., the dorsal and ventral premotor cortex and the frontal eye fields, columns of frontal terminals were most prominent in the convexity of cortex between the superior and inferior limbs of the arcuate sulcus, whereas parietal columns were most numerous in the banks and depths of the superior and inferior sulci (Figs. 2, 3). Columns of prefrontal and parietal terminal fields also interdigitated in the premotor cortex just ventral to the inferior arcuate sulcus (Fig. 2). In the orbital prefrontal cortex between the lateral and medial orbital sulci, a single column or cluster of columns originating from the prefrontal cortex was flanked by adjacent parietal columns (Fig. 2). The projection of the posterior parietal cortex to the caudomedial lobule was limited to a single column of terminals that adjoined a column of prefrontal terminals (Figs. 2, 7B).

Laminar complementarity of prefrontal and parietal terminal fields. A completely different pattern was noted in prefrontal and parietal projections to the frontoparietal operculum and to the superior temporal cortex (Figs. 2, 5). In these areas, terminal labeling from the 2 cortices was present in the same columns or cluster of adjacent columns but exhibited a laminar complementarity. In both regions, prefrontal terminal labeling was concentrated in layer I, with a moderate distribution in layers III and $\mathrm{V}$, whereas parietal terminals occupied mainly layer IV but also layer VI directly beneath the prefrontal terminal labeling. It is interesting to note, however, that faint columns of terminal labeling were superimposed on the more salient pattern of laminar alternation. Dense clusters of prefrontal label in layers III and $\mathrm{V}$ were aligned within a vertical column and often located adjacent to dense clusters of parietal terminals in layers IV and VI. Furthermore, within "parietal" columns prefrontal terminal labeling appeared to be increased above background levels in layers IV and VI, and faint parietal terminal labeling was present in layers I, III, and V of adjacent "prefrontal" columns. This same laminar specificity was evident in the contralateral hemisphere, though only the layers most densely labeled in the ipsilateral hemisphere were innervated contralaterally. For example, in the hemisphere opposite to the injection sites in case 3 , prefrontal terminal labeling was restricted to layer $I$ in the fundus of the sulcus, while parietal terminals were confined to layer IV.

In summary, 2 distinct patterns of termination were observed in the common cortical projections of the prefrontal and parietal cortices (Fig. 8). In certain cortical target areas, prefrontal and parietal terminal fields formed an array of interdigitating columns, while in other areas, prefrontal and parietal projections converged on the same column or cluster of adjacent columns but terminated within different laminae. However, exceptions to these 2 general patterns were found. For example, a variant of the laminar patterning found in the frontoparietal operculum and superior temporal sulcus was observed in the insular cortex (Figs. 2; 9, $C, D$ ). Prefrontal terminals were located in layers I, III, and V, while the parietal projection terminated in all layers but was least dense in layer I. Similarly, in the parahippocampal cortex (area TF), prefrontal terminals were concentrated in layer I, whereas parietal terminals were located in deeper layers (III, V , and VI) (Fig. 2). Finally, the terminal patterning of the projections of the prefrontal and parietal cortices to the presubiculum differed from that observed in any other cortical area. In the presubiculum, both prefrontal and parietal terminals were present in layer I of the very same region of the posterior presubiculum; therefore, extensive overlap of prefrontal and parietal terminals was observed in this cortical area (Figs. 2, 7A).

\section{Subcortical targets}

Prefrontal and parietal terminal fields were observed in close apposition in several subcortical areas (Fig. 2). These included the neostriatum, claustrum, thalamus, superior colliculus, and brain-stem reticular formation. Here again, it should be noted that this list of subcortical target areas may not be exhaustive. Particularly at brain-stem levels, where individual nuclei are small, dual innervation of some sites may have been missed since only every 5 th or 10 th section was collected through this region. While few nonconvergent projections were observed at the subcortical level, one noteworthy exception is the projection of the prefrontal cortex to the mesencephalic central gray, an area that apparently does not receive cortical input from the posterior parietal cortex (Arnsten and Goldman-Rakic, 1984).

Neostriatum. The neostriatal projections of the prefrontal and parietal cortices have been described in detail previously (Selemon and Goldman-Rakic, 1985) and therefore will not be repeated here. In the present context, the main finding was that prefrontal and parietal cortices project to adjacent territories within the neostriatum throughout the longitudinal extent of this subcortical structure (Figs. 2; 9, $A, B$ ).

Claustrum. Prefrontal and parietal projections terminated throughout the entire A-P extent of the claustrum (Fig. 2); how- 
INIERDIGITATION MODE

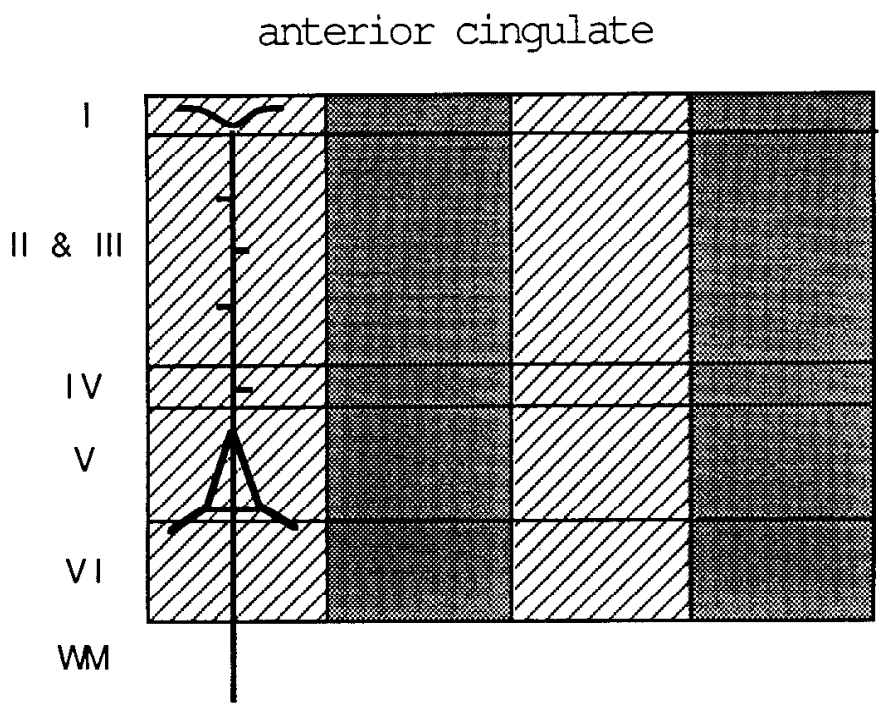

COMPLEMENTARY LAYERS MODE

superior temporal sulcus

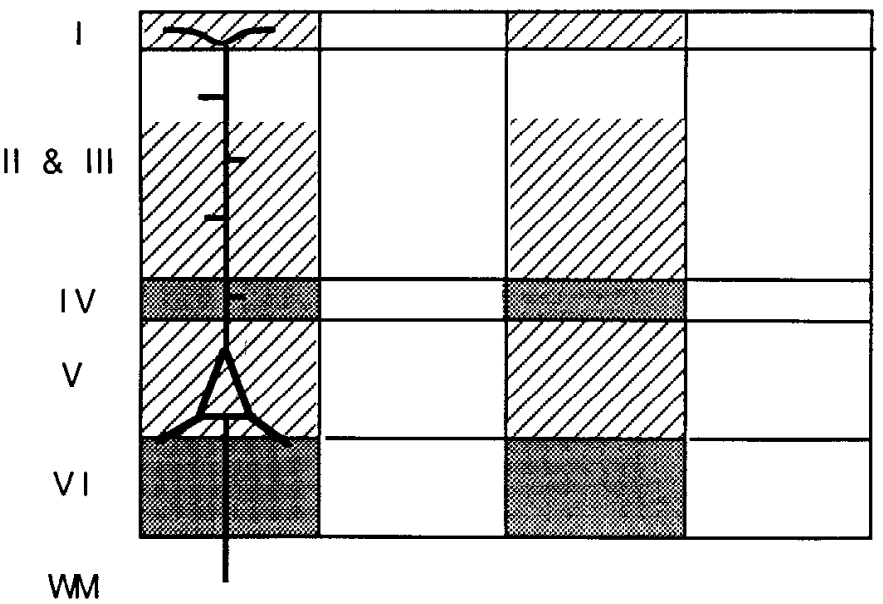

prefrontal
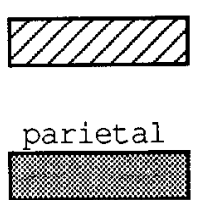

Figure 8. Schematic illustration of the 2 patterns of cortical termination observed in the present study. In most cortical regions (e.g., in the anterior cingulate cortex), prefrontal and parietal terminal labeling forms an interdigitating array of columns. A second, less common pattern was observed (e.g., in the superior temporal sulcus) in which prefrontal terminals occupy layers I, III, and $\mathrm{V}$ with parietal terminals filling complementary layers IV and VI. ever, at anterior levels of the claustrum the relationships of these 2 terminal fields was obscured by the presence of large numbers of prefrontal fiber bundles. More posteriorly within the claustrum, prefrontal and parietal terminal fields were adjacent within the ventral portion of the nucleus (Figs. $2 ; 9, C, D ; 10$ ). In some paired sections the parietal terminal field appeared to be interposed between dorsally and ventrally adjacent territories of prefrontal terminal labeling (Fig. 9, $C, D$ ).

Amygdala. A weak projection from the parietal injection site to the basolateral nucleus of the amygdala was observed in case 2 , and a prefrontal projection to this same nucleus was found in case 3 . Therefore, although projections from prefrontal and parietal cortices to the amygdala were not seen in the same animal, it seems likely that the basolateral nucleus of the amygdala is a common efferent target of these areas.

Thalamus. In many respects the interrelationship of prefrontal and parietal projections in the thalamus resembled that observed in the neostriatum. In the thalamus, prefrontal and parietal projections occupied adjacent territories that extended throughout virtually the entire A-P expanse of the thalamus with very little overlap between parietal and prefrontal fields (Figs. 2, 11). Moreover, the relative size of the 2 projections shifted such that the prefrontal projection was much larger than the parietal projection at rostral levels of the thalamus, and the reverse was true caudally in the thalamus.

In the rostral thalamus, the prefrontal cortex projected bilaterally to the anterior medial (AM) and midline nuclei (Fig. 11). The prefrontal projection to AM formed a continuous, dorsally located band that extended from the lateral border of AM across the midline to the contralateral border zone as reported previously (Goldman, 1979; Preuss and Goldman-Rakic, 1987). In contrast, the parietal projection to AM was situated more ventrally within AM, had a patchy appearance and was largely restricted to the ipsilateral hemisphere. The prefrontal projection to the midline nuclei consisted of 2-4 discrete patches of terminal label. The parietal projection to AM extended into the ventrally adjacent midline nuclei, where clusters of prefrontal and parietal terminals appeared to avoid one another. The pre- 

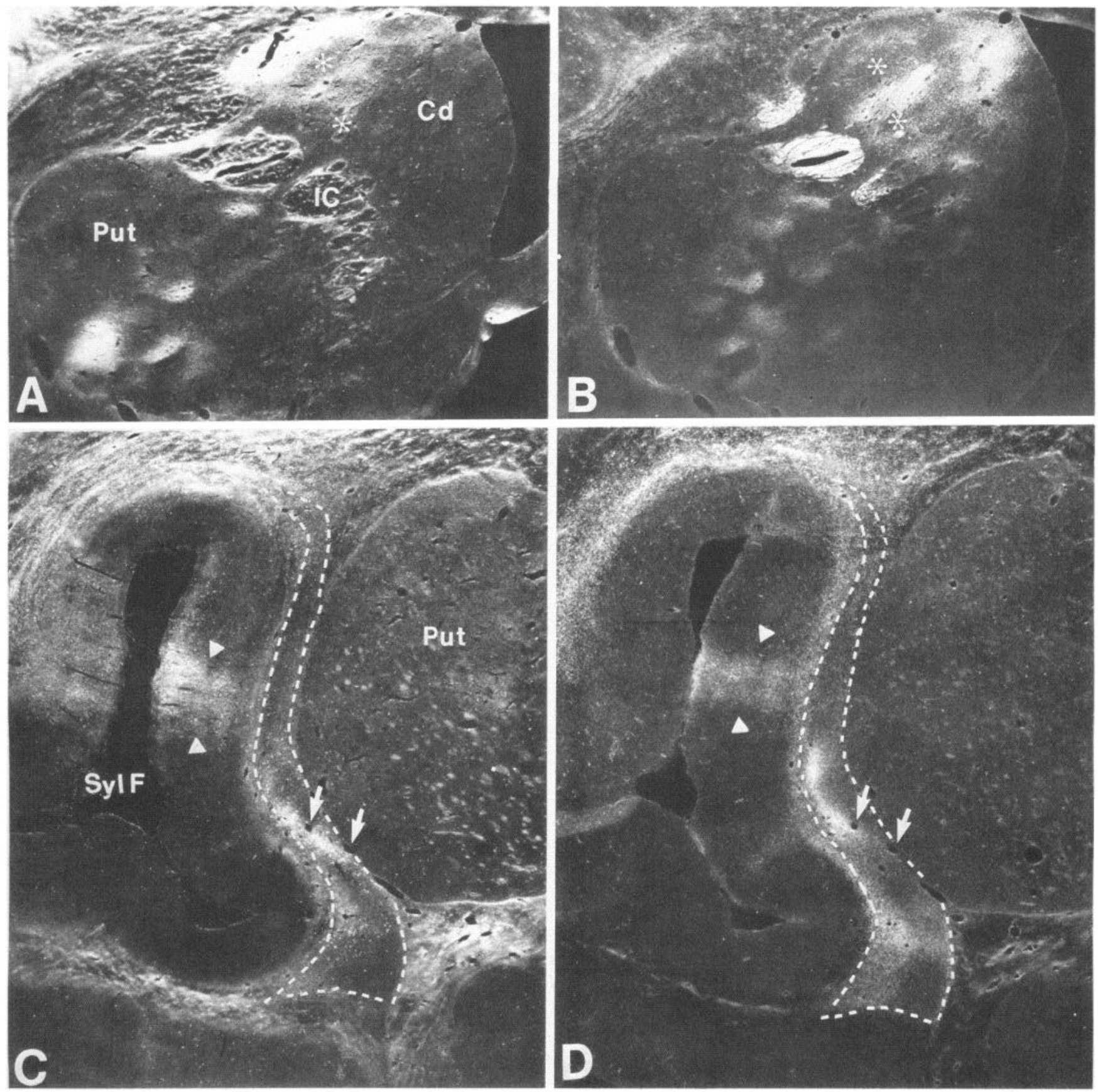

Figure 9. Projections of the prefrontal and parietal cortices to the neostriatum, claustrum, and insular cortex in case 2 . $A$, In the rostral neostriatum, parietal terminal labeling is located in the dorsolateral caudate nucleus and extends ventrally into the medial rostral putamen. $B$, Prefrontal terminal labeling is located more medially within the rostral caudate and ventrally adjacent putamen. $C$, Parietal terminal labeling within the claustrum, as well as a single column of label in the insular cortex, are shown. $D$, In an adjacent section, 2 clusters of prefrontal terminal labeling in the claustrum are present; comparison of the 2 sections reveals that the prefrontal projection borders the parietal terminal field dorsally and ventrally. A single column of prefrontal terminals overlaps the parietal column in the insular cortex; however, prefrontal terminal labeling is primarily located in layer I, the layer in which parietal labeling is sparse. Arrows and arrowheads point to the same blood vessels in the claustrum and insular cortex, respectively, to facilitate comparison of adjacent sections. Abbreviations: $C d$, caudate; $I C$, internal capsule; $P u t$, putamen; $S y l F$, Sylvian fissure. Magnification: $A$ and $B, \times 6 ; C$ and $D, \times 5$.

frontal and parietal projections also were coextensive throughout the medial dorsal (MD) and lateral dorsal (LD) nuclei (Figs. $2,11)$. While the prefrontal projection densely innervated the parvicellular subdivision of MD, parietal terminals formed a thin arc at the lateral border of MD. A few clusters of parietal terminals extended medially into the prefrontal domain where they appeared to fit into holes or fenestrations in the prefrontal projection (Figs. $2 ; 12, A, B$ ). In $\mathrm{LD}$, the prefrontal terminal field was located dorsomedially; parietal terminal labeling was situated ventromedially (Figs. 2, 11). Terminal labeling from both cortices was present dorsally within the ventrolateral (VL) nucleus (Figs. 2, 11). Prefrontal terminal labeling was restricted to a narrow strip of labeling at the dorsal margin of the nucleus adjacent to the capsule surrounding $\mathrm{LD}$, whereas parietal ter- 


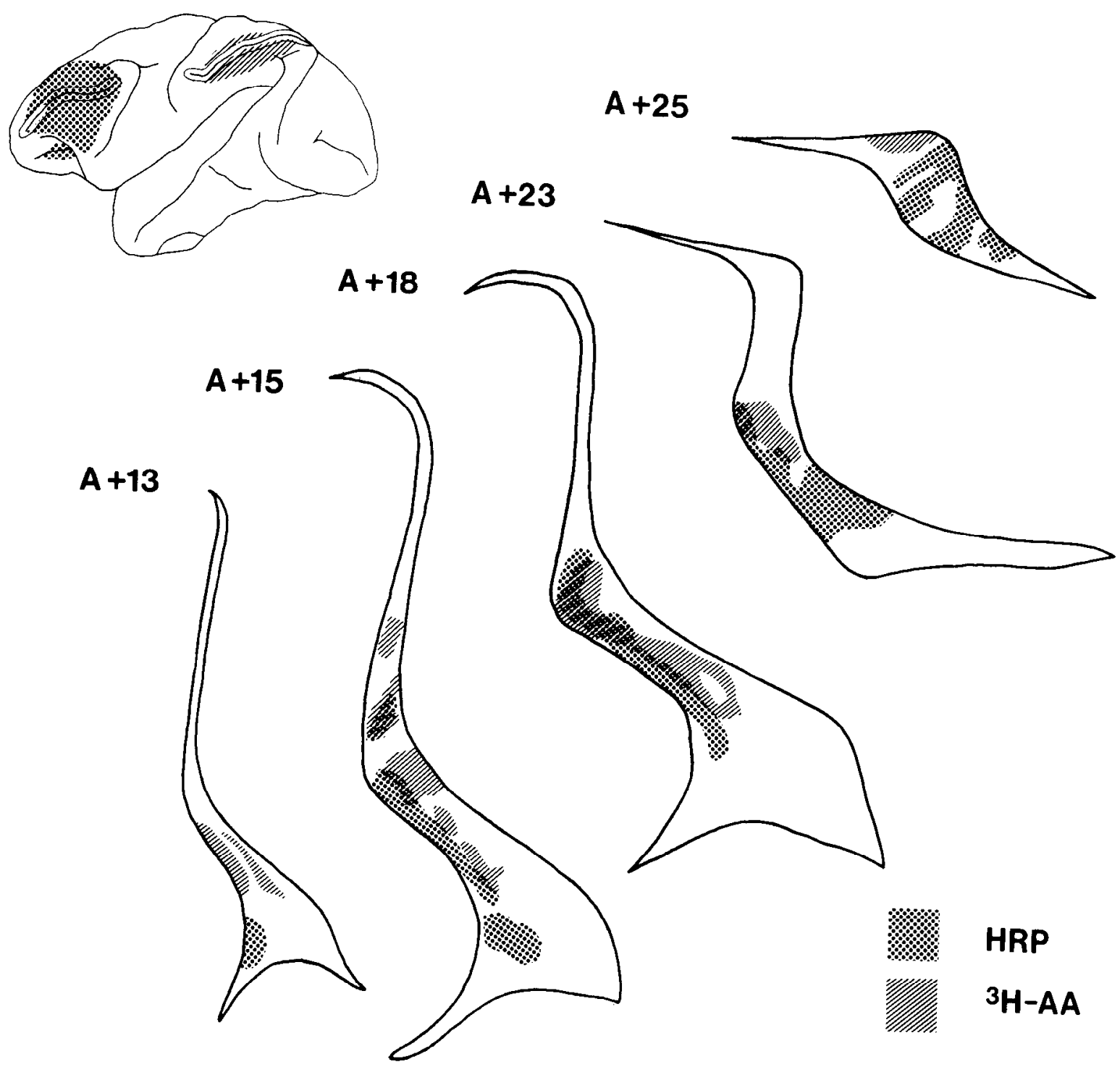

Figure 10. Drawing of the superimposed projections of the prefrontal and parietal cortices to the claustrum in case 3. Parietal (cross-hatching) and prefrontal (stippling) terminal fields are shown at 5 rostrocaudal levels $(A+25-A+13)$ of the claustrum. At rostral levels, parietal terminal labeling is located lateral to the prefrontal projection. At more caudal levels, the parietal field shifts to a position dorsal to the prefrontal terminal zone.

minal labeling was more diffusely distributed over a wide dorsal band of VL. Prefrontal and parietal projection zones were apposed throughout the medial pulvinar nucleus as well (Figs. 2; $11 ; 12, C, D)$. The large parietal projection occupied a dorsal territory within the lateral part of the medial pulvinar. The much smaller prefrontal projection was contiguous with the medial edge of the parietal terminal field at all A-P levels of the nucleus.

Superior colliculus. The intermediate layers of the superior colliculus received projections from the prefrontal and parietal cortices in cases 1 and 3 (Figs. 2, 13). Both projections exhibited a discontinous patterning such that approximately 4 patches of prefrontal terminal labeling and 4 or 5 patches of parietal terminal labeling were present in any given coronal section. While the relationship between the prefrontal and parietal patches was not clear-cut, there was some indication of interdigitation of the 2 terminal fields.

Pontine reticular formation. In case 3, prefrontal terminals were found within the central superior nucleus of the reticular formation just dorsal to the parietal projection to the ventral tegmental nucleus (Fig. 2).

\section{Discussion}

The dorsolateral prefrontal cortex and the posterior parietal cortex project to at least 15 distinct areas of the ipsilateral cortex (see Summary diagram, Fig. 14), to at least 3 contralateral cortical areas and to many of the same subcortical targets as well. The existence of an extensive common efferent circuitry suggests that the prefrontal and parictal corticcs are part of a larger neural system that mediates behavior in a specific cognitive domain. At the cortical level this neural network includes premotor centers, in particular the frontal eye fields and supplementary motor cortex, limbic areas, other association cortices, and a region of the somatosensory cortex in the frontal-parietal operculum. Prominent subcortical areas that receive both prefrontal and parietal inputs are the neostriatum, thalamus, and claustrum at telencephalic levels and the superior colliculus and pontine re- 


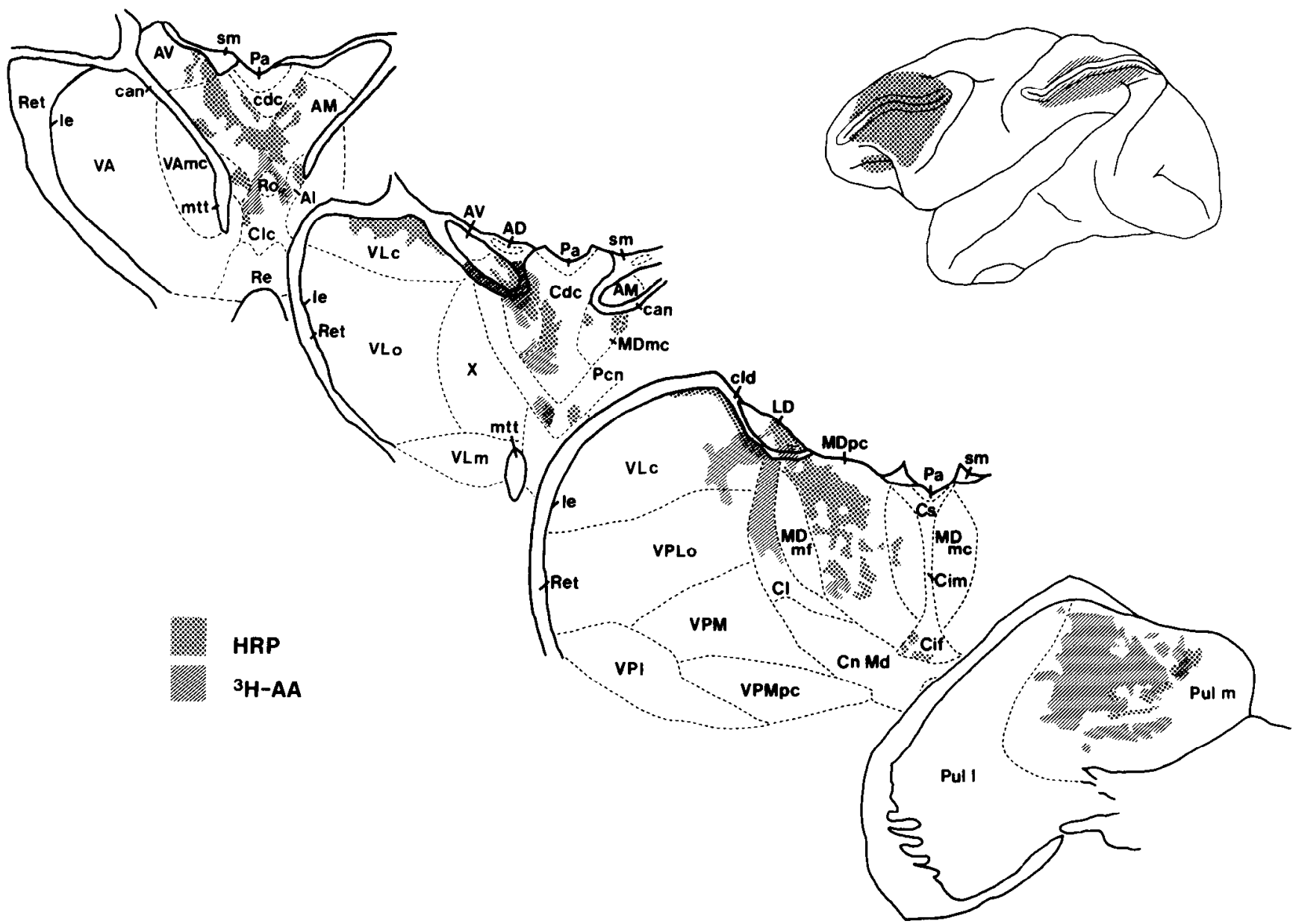

Figure 11. Corticothalamic projections from the prefrontal and parietal cortices at 4 rostrocaudal levels of the thalamus in case 3. At rostral levels, the prefrontal projection is much larger in size than the more ventrally located projection of the parietal cortex. At caudal levels, the parietal projection enlarges and moves to lie lateral to the smaller prefrontal terminal field. Abbreviations: $A D$, anterior dorsal nucleus; $A l$, alaris; $A M$, anterior medial nucleus; $A V$, anterior ventral nucleus; $c a n$, capsule of the anterior nuclei; $C d c$, central nucleus, pars densocellularis; $C l c$, central nucleus, pars latocellularis; $C l$, centrolateral nucleus; $C i f$, central nucleus, pars inferior; $C i m$, central nucleus, pars intermedialis; $C s$, central nucleus, pars superior; $C n M d$, centromedian nucleus; $L D$, lateral dorsal nucleus; le, lamina externa; mtt, mammillothalamic tract; $M D m f$, medial dorsal nucleus, pars multiformis; $M D m c$, medial dorsal nucleus, pars magnocellularis; $M D p c$, medial dorsal nucleus, pars parvicellularis; $P a$, paraventricular nucleus; $P c n$, paracentral nucleus; $P u l m$, medial pulvinar nucleus; $P u l l$, lateral pulvinar nucleus; $R e$, nucleus reuniens; $R e t$, reticular nucleus; $R o$, nucleus rotundus; $s m$, stria medullaris; $V A$, ventral anterior nucleus; $V A m c$, ventral anterior nucleus, pars magnocellularis; $V L c$, ventral lateral nucleus, pars caudalis; VLm, ventral lateral nucleus, pars medialis; VLo, ventral lateral nucleus, pars oralis; VPI, ventroposterior inferior nucleus; $V P L o$, ventroposterior lateral nucleus, pars oralis; $V P M$, ventroposterior medial nucleus; $V P M p c$, ventroposterior medial nucleus, pars parvicellularis $X$, area $\mathrm{X}$.

ticular formation in the brain stem. While it is relatively easy to speculate that prefrontal and parietal input to the frontal eye fields and superior colliculus may direct eye movements to spatially coded targets, it is more difficult to assign a specific function to each of the other areas. However, the present findings suggest that areas such as the superior temporal sulcus may play a role in spatially guided behavior: Physiologic study of these regions will provide further insight into their functional relationship to the dorsolatcral prefrontal and posterior parietal cortices.

\section{Convergence of prefrontal and parietal terminal fields}

Double labeling of prefrontal and parietal projections in the same monkey enabled us to examine the precise relationship of the 2 terminal fields. In virtually all areas examined, prefrontal and parietal terminal fields occupied distinct territories. Overlap of prefrontal and parietal projections was much less prevalent than segregation in all common cortical and subcortical sites with the exception of the presubiculum. The segregation of prefrontal and parietal terminal fields suggests that the brain utilizes the spatial dimension to code incoming information. At the cortical level, prefrontal and parietal projections were segregated either vertically into spatially distinct columns of terminal labeling or horizontally into different cortical laminae within a series of columns. Subcortically, prefrontal and parietal terminals most often were located in adjacent, longitudinally extensive territories within a given nucleus, as in the neostriatum, claustrum, and pulvinar nucleus of the thalamus but in some regions were located in adjoining nuclei as in the pontine reticular formation and LD/VL nuclei of the thalamus. Thus, at the subcortical level, the pattern of prefrontal and parietal termination suggests that the 2 cortices project in apposition to most subcortical targets. The closeness of their efferent terminations is all the more impressive because of the wide separation be- 

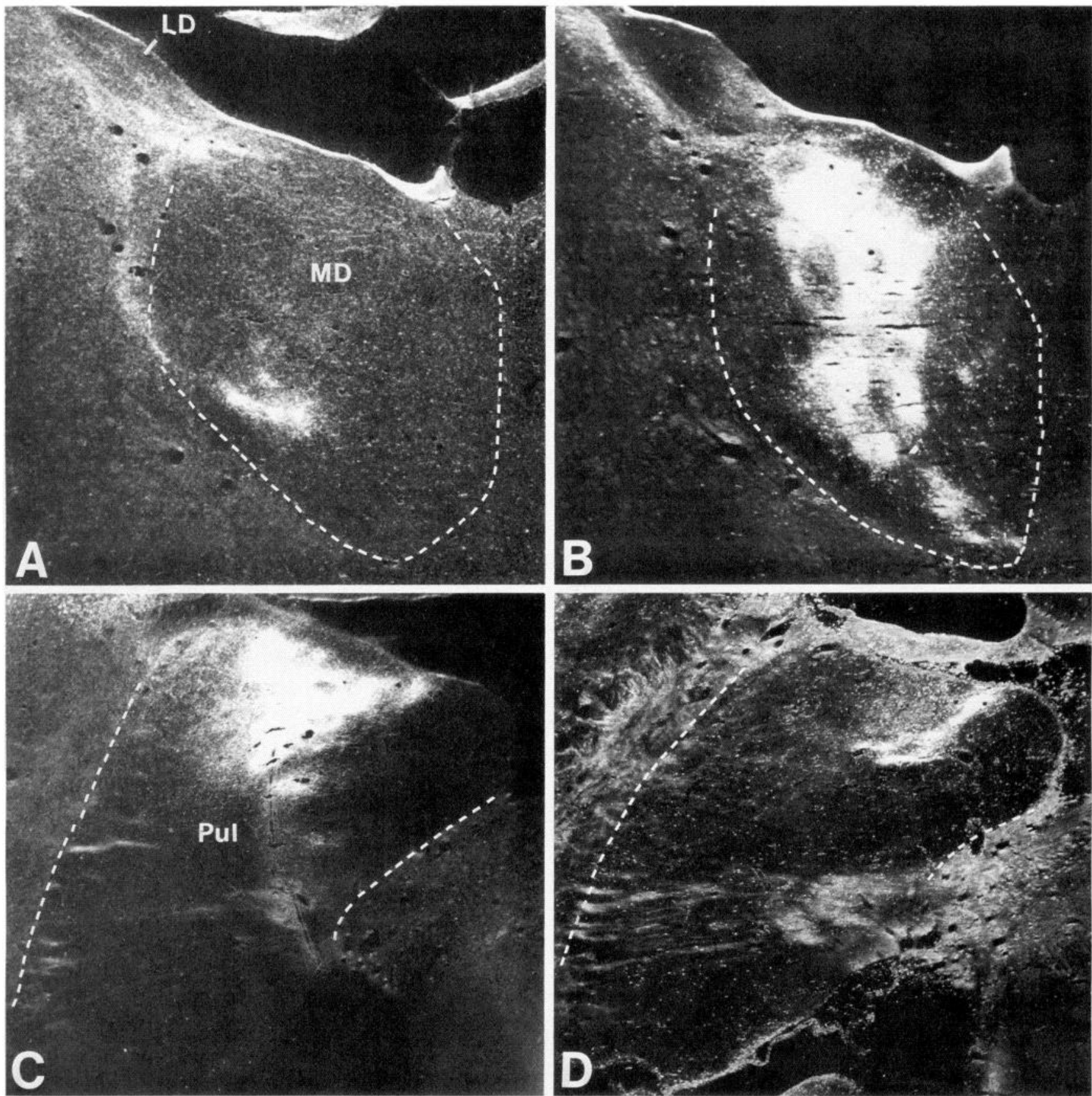

Figure 12. Dark-field photomicrographs illustrating the projection of the prefrontal and parietal cortices to the medial dorsal and medial pulvinar nuclei of the thalamus in case 3. $A$, Parietal terminals are situated at the lateral border of the medial dorsal nucleus with a few patches of label found ventrally within the nucleus. $B$, The prefrontal projection nearly fills the parvicellular division of the medial dorsal nucleus, although unlabeled holes are present in the otherwise dense terminal plexus. In $C$, the parietal projection to the pulvinar nucleus occupies an extensive territory within the lateral portion of the medial pulvinar nucleus, whereas in $D$, the prefrontal terminal field is much smaller and more medially situated within the pulvinar. Abbreviations: $L D$, lateral dorsal nucleus; $M D$, medial dorsal nucleus; $P u l$, pulvinar. Magnification: $A$ and $B, \times 15.4 ; C$ and $D, \times 9.25$.

tween prefrontal and parietal cortices on the cerebral surface. Moreover, there were subcortical nuclei in which some hint of interdigitation of prefrontal and parietal projections was observed: These were the claustrum, the MD nucleus of the thalamus, where small patches of parietal terminals appeared to fill holes in the much larger prefrontal projection, and in the superior colliculus, where patches of prefrontal and parietal terminals were aligned along the mediolateral axis of the colliculus. Convergence in the strict sense of the term, that is, termination of 2 inputs of the same neuron, would seem to be rather limited in the case of prefrontal and parietal inputs. Whether prefrontal and parietal projections terminate on the same neuron at the cortical level will remain uncertain until appropriate ultrastructural data are available. At least in those regions where prefrontal and parietal terminals occupy different laminae within a vertical column, it seems highly probable that the 2 inputs converge onto different portions of the dendritic trees of pyramidal neurons (Fig. 8), much as hippocampal inputs are distributed on 

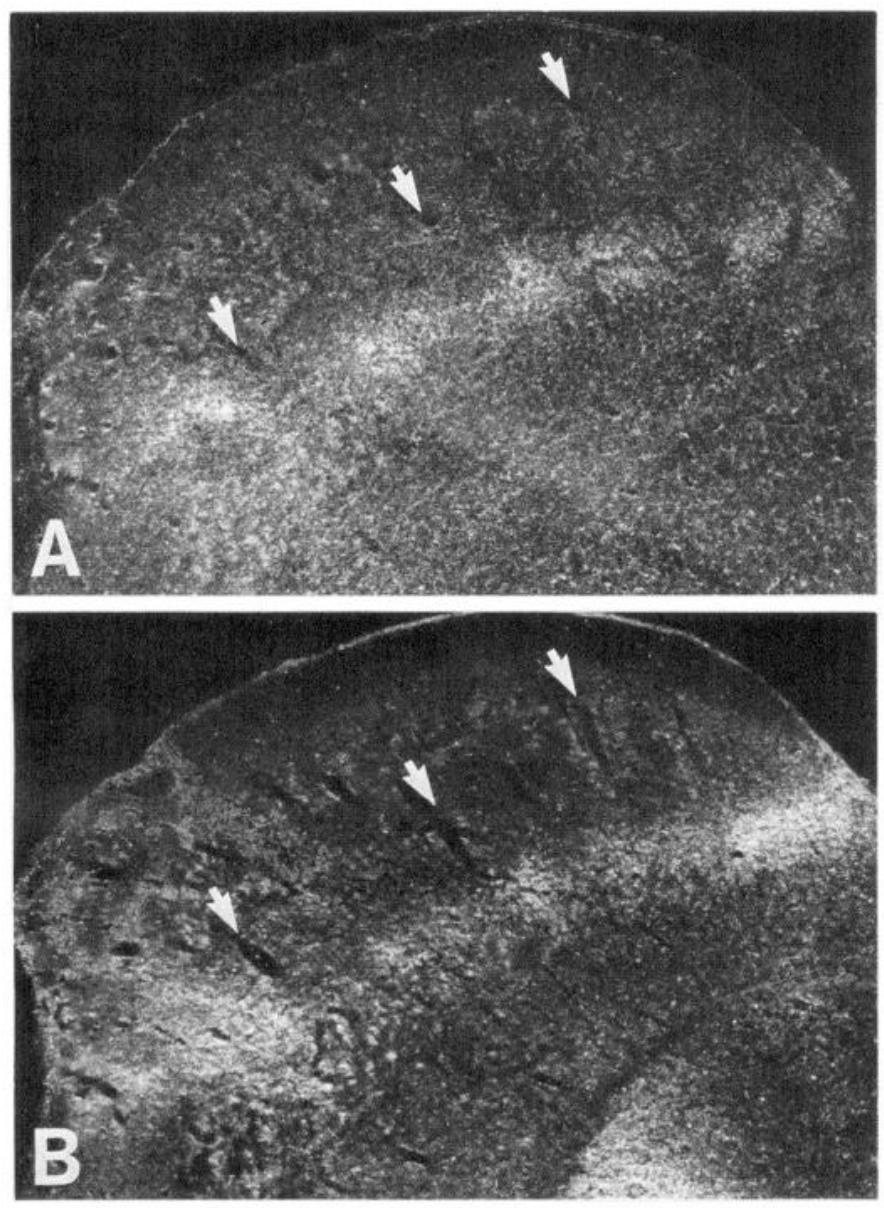

Figure 13. Dark-field photomicrographs of the superior colliculus in case 3 . The projections of the prefrontal $(A)$ and parietal cortices $(B)$ to the intermediate layers of the colliculus are shown. Patches of prefrontal and parietal terminals span the medial-lateral axis of the intermediate layers. Arrows point to the same 3 blood vessels in these adjacent sections. $\times 18.8$.

proximal and distal segments of pyramidal neurons in Ammons horn.

\section{Dual patterns of prefrontal-parietal termination in the cortex}

In many cortical target areas, prefrontal and parietal projections terminate in adjacent, spatially alternating columns. Columnar interdigitation of cortical terminal fields was originally described for association and callosal inputs to the principal sulcal cortex (Goldman-Rakic and Schwartz, 1982). In areas receiving columnar projections, prefrontal and parietal terminals contact primarily separate sets of neurons, that is, neurons in adjacent columns. A very different pattern of interaction is found in the frontoparietal operculum and the superior temporal sulcus where prefrontal and parietal fields are located in alternating laminae within the same column. The presence of 2 distinct patterns of cortical termination suggests that the integration of prefrontal and parietal information differs accordingly. Perhaps, prefrontal and parietal inputs act competitively at sites in which they occupy adjacent columns such that, for example, either the prefrontal or parietal cortex may activate the cingulate cortex at any given moment. An analogous situation may exist in the neostriatum, where cortical access to the motor system may be gated in part by having adjacent terminal domains inhibit one another (Groves, 1983). In contrast, prefrontal and parietal inputs to different laminae within a single column may interact cooperatively to reinforce a particular perception or affect a behavior. For instance, the cortex in the depths of the superior temporal sulcus may be an area in which incoming spatial information from the parietal cortex is compared and summated with an internal trace of the spatial world supplied by the frontal input. While the significance of these 2 patterns of termination is obscure at this time, the existence of diverse anatomical relationships must in some manner reflect a duality of cortical processing mechanisms.

\section{Prefrontal-parietal efferent circuitry in relation to forward/ feedback schema}

Recent analyses of cortical pathways in the visual system have shown that forward and feedback projections have distinct laminar patterns of termination: that is, forward projections terminate primarily in layer IV, whereas feedback pathways terminate mainly in layer I (Rockland and Pandya, 1979; van Essen and Maunsell, 1983). Many of the prefrontal and parietal projections described in the present study do not fit either classification because they terminate in a columnar fashion extending throughout all 6 layers, though possibly terminating in different densities in different layers. While we have considered the possibility that columnar patterning might actually represent labeling of fibers of passage en route to their terminal destination in layer I, the presence of clear-cut laminar patterning, as for example in the projection of the prefrontal cortex to layers I, III, and V of the superior temporal cortex, illustrates that fiber labeling does not obscure discrete terminal labeling within specific layers. In target areas that exhibit discrete laminar patterning, such as the frontoparietal operculum and superior temporal cortex, the forward and feedback designations may be applicable. The parietal projections to these areas terminate primarily in layer IV and as such are analogous to forward pathways of the visual system; the prefrontal projections terminate largely in layers I, III, and V and therefore have a laminar patterning that may be considered feedback. Thus, the laminar specificity of prefrontal and parietal terminations may give some indication of the direction of information flow in the cortical circuitry involved in spatial perception. Accordingly, one might suppose that the parietal cortex relays visual information that is relevant for spatial perception to temporal and opercular cortices, which in turn relay spatial percepts to the prefrontal cortex. The prefrontal cortex, which receives spatial information directly via its corticocortical connections with the parietal cortex and indirectly via relays in the temporal and opercular cortices, feeds highly processed information back to all of these cortices. Perhaps in regions like the superior temporal cortex, input from the prefrontal cortex represents an internal memory trace for spatial information which could be compared against incoming, spatial coordinates from the external world via the parietal cortex.

Previous studies of cortical connectivity have suggested that the prefrontal cortex is the final destination and point of convergence for multisynaptic relays from the primary visual, auditory, and somatosensory cortices (Pandya and Kuypers, 1969; Jones and Powell, 1970; Chavis and Pandya, 1976). It is interesting in this regard that prefrontal projections throughout the cortex terminate in layer I; even in regions innervated by a columnar prefrontal projection, terminal density appears to be 


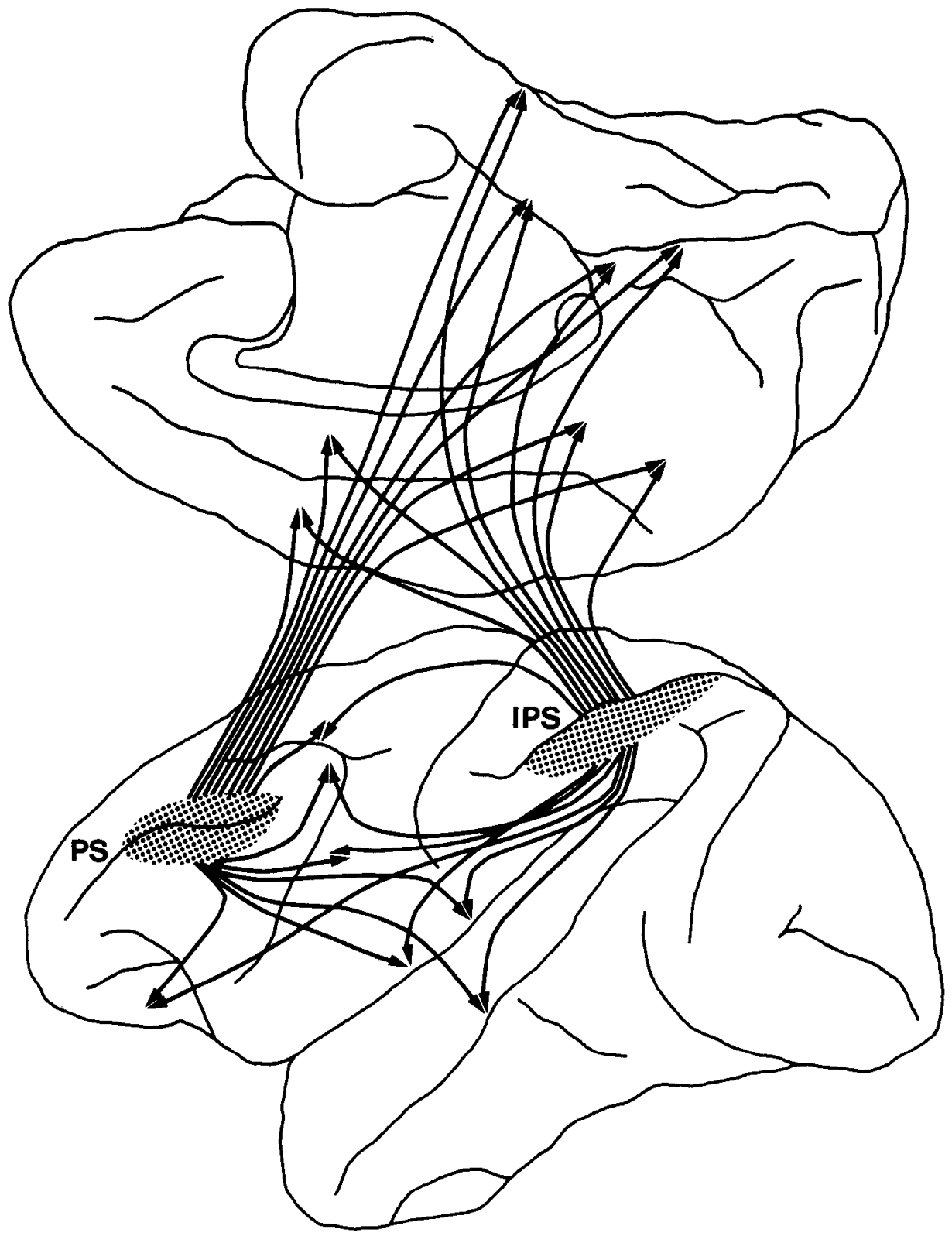

Figure 14. Schematic summary of the common cortical targets of the dorsolateral prefrontal and posterior parietal cortices. The targets depicted are the orbitofrontal cortex, premotor areas inclusive of the supplementary motor area, the frontal eye fields, the anterior and posterior cingulate cortices, the frontoparietal operculum, the insula, the superior temporal sulcus, the medial parietal cortex, the parahippocampal and presubicular cortices, the caudomedial lobule, and the medial prestriate cortex. $I P S$, intraparietal sulcus; $P S$, principal sulcus. greatest in layer I. This suggests that prefrontal projections are relaying highly processed information to areas in which information processing is more closely attuned to primary sensory perceptions. Thus, in terms of cortical hierarchy, the present findings are consistent with the notion that the prefrontal cortex represents the apex of the pyramid, with all other areas feeding information forward to and receiving feedback projections from the prefrontal cortex.

\section{Prefrontal-parietal efferent circuitry: a distributed neural network}

Classically, the brain was thought to be composed of functionally specialized centers, with each brain center mediating a unitary function distinct from that subserved by all other centers. However, the concept of focal centers of function does not explain the clinical observation that lesions in many different areas of the brain produce similar deficits. More contemporary views of functional localization in the brain suggest that several brain centers may be interconnected in a network such that function is distributed among the interconnected areas. We propose that the elaborate network of efferent connections uncovered in the present study represents a neural circuit that is functionally specialized for spatially guided behavior. This does not imply functional redundancy but rather indicates that each link in the circuit mediates a single aspect of the multifaceted processing involved in spatially related behavior. Perception of the spatial dimension probably is relegated to the occipital and superior temporal cortices, while the cingulate and limbic cortices may attach emotional significance or coloring to spatial percepts. Certainly, spatially dependent movement would involve premotor cortices, the basal ganglia, and superior colliculus. Interposed between perceptual and motor portions of the circuit are the parietal and prefrontal association cortices. Both of these areas orchestrate spatially related behavior; yet even at this level there may be division of labor such that the parietal cortex constructs spatial coordinates and maps from incoming visual 
information (Mountcastle et al., 1984), while the dorsolateral prefrontal cortex accesses these representations to govern behavior requiring memory of spatial position (Goldman-Rakic, 1987).

Recently, Pandya and Yeterian (1984) described a neural circuit for spatial perception with the posterior parietal cortex at the hub sending efferents to many different areas of the cortex. The present study, which has shown that the prefrontal cortex projects to virtually all of these same cortical areas, reveals the parallel role that the prefrontal cortex plays in spatially related behavior. Moreover, the present study shows that the parietal and prefrontal cortices innervate an array of subcortical target areas which should be included in this functionally specialized circuit. On the basis of parietal afferent circuitry, Mesulam (1981) proposed that the parietal, frontal eyefields, and cingulate cortices, in conjunction with subcortical reticular areas, form a network that controls directed attention. While the connections described in the present study are efferent, it should be noted that with the exception of the presubiculum, all the cortical projections we observed were reciprocal. Therefore, the circuitry we have uncovered represents a much broader and reciprocal network in which the prefrontal association cortex is a prominent and integral part. Furthermore, we suggest that this network is involved in all aspects of spatial perception and behavior, including attention, perception, memory, and motor control (Goldman-Rakic, 1987, 1988).

\section{Parallel subsystems within the prefrontal-parietal efferent network}

A recent study from our laboratory has shown that each cytoarchitectonic subdivision of the posterior parietal cortex is reciprocally connected with a distinct region of the prefrontal cortex surrounding the principal sulcus (Cavada and GoldmanRakic, 1985). This finding raises the possibility that there is a comparable parcellation of the common efferent circuitry. In support of this hypothesis, Cavada and Goldman-Rakic (1986) have shown that, while each of the subdivisions of the parietal cortex (areas $7 \mathrm{a}, 7 \mathrm{~b}, 7 \mathrm{ip}$, and $7 \mathrm{~m}$ ) project to a dozen or more of the common efferent targets, each terminates in a different part of the target area. A few target areas do not receive input from all subdivisions of the posterior parietal cortex. For example, the frontoparietal operculum receives parietal input specifically from area $7 \mathrm{~b}$ (Petrides and Pandya, 1984; Cavada and Goldman-Rakic, 1986), whereas the superior temporal sulcus (area IPa of Seltzer and Pandya, 1978) is innervated mainly by areas 7a and 7ip (Seltzer and Pandya, 1984; Cavada and Goldman-Rakic, 1986); likewise, the parahippocampal and presubicular cortices are innervated mainly by area $7 \mathrm{a}$ and not by area $7 \mathrm{~b}$ (Seltzer and Van Hoesen, 1979; Cavada and GoldmanRakic, 1986). However, areas 7a, 7b, and 7ip project independently to a distinct subregion of every other target area described in the present study. Therefore, it appears that areas $7 \mathrm{a}, 7 \mathrm{~b}$, and 7 ip are each part of a large network of interconnected areas and that the separate nctworks may be organized in parallcl. Additional experiments in which small amounts of tracers are placed in reciprocally connected areas of the parietal and prefrontal cortices are needed to determine with certainty that interconnected areas project to the same topographic zone within any given target region. If this were so, it might be more accurate to think of the circuitry involved in spatial perception as a set of parallel circuits, each controlling a particular aspect of spatial perception or spatially guided behavior.

\section{Functional pairing of pre- and post-Rolandic cortices}

The present findings show that 2 widely separated areas of cortex are tightly linked throughout the neural circuit which governs spatial perception and behavior. Whether other areas of the prefrontal cortex are linked with post-Rolandic cortices in mediating different functions remains to be seen. However, Goldman-Rakic $(1987,1988)$ recently has speculated that this may be true: She proposes that the prefrontal cortex as a whole performs a unitary function, that of holding information gathered by the more posterior cortices "on-line" in short-term memory. Thus, while the dorsolateral prefrontal and posterior parietal cortices may be paired via reciprocal corticocortical connections (and via common outflow pathways) in mediating visuospatial function, the orbitofrontal and inferotemporal cortices may be linked in an analogous circuit to govern auditory-related behavior and behavior dependent on detection of specific features of objects, such as their size and shape. Additional doublelabeling studies are required to determine whether other prefrontal areas are linked with post-Rolandic cortices via a network of common efferent targets. If so, the prefrontal-parietal network described here may be paradigmatic of a large number of functionally diverse neural circuits.

\section{References}

Aggleton, J. P., M. J. Burton, and R. E. Passingham (1980) Cortical and subcortical afferents to the amygdala of the rhesus monkey $(\mathrm{Ma}$ caca mulatta). Brain Res. 190: 347-368.

Andersen, R. A., G. K. Essick, and R. M. Siegel (1985) Encoding of spatial location by posterior parietal neurons. Science 230:456-458.

Arnsten, A. F. T., and P. S. Goldman-Rakic (1984) Selective prefrontal cortical projections to the region of the locus coeruleus and raphe nuclei in the rhesus monkey. Brain Res. 306: 9-18.

Asanuma, C., R. A. Andersen, and W. M. Cowan (1985) The thalamic relations of the caudal inferior parietal lobule and the lateral prefrontal cortex in monkeys: Divergent cortical projections from cell clusters in the medial pulvinar nucleus. J. Comp. Neurol. 241: 357-381.

Baleydier, C., and F. Mauguiere (1980) The duality of the cingulate gyrus in monkey. Neuroanatomical study and functional hypothesis. Brain 103: 525-554.

Barbas, H., and M.-M. Mesulam (1981) Organization of afferent input to subdivisions of area 8 in rhesus monkeys. J. Comp. Neurol. 200: 407-431.

Barbas, H., and D. N. Pandya (1987) Architecture and frontal cortical connections of the premotor cortex (area 6 ) in the rhesus monkey. J. Comp. Neurol. 256: 211-228.

Brodal, P. (1980) The cortical projection to the nucleus reticularis tegmenti pontis in the rhesus monkey. Exp. Brain Res. 38: 19-27.

Cavada, C., and P. S. Goldman-Rakic (1985) Paricto-prefrontal connections in the monkey: Topographic distribution within the prefrontal cortex of sectors connected with the lateral and medial parietal cortex. Soc. Neurosci. Abstr. 11: 323.

Cavada, C., and P. S. Goldman-Rakic (1986) Subdivisions of area 7 in the rhesus monkey exhibit selective patterns of connectivity with limbic, visual and somatosensory cortical areas. Soc. Neurosci. Abstr. 12: 262.

Chavis, D. A., and D. N. Pandya (1976) Further observations on corticofrontal connections in the rhesus monkey. Brain Res. 11:369386.

Critchley, M. (1953) The Parietal Lobe, Edward Arnold, London.

Denny-Brown, D., and B. Q. Banker (1952) Amorphosynthesis from left parietal lesion. Arch. Neurol. Psychiatry 71: 302-313.

Funahashi, S., C. J. Bruce, and P. S. Goldman-Rakic (1986) Perimetry of spatial memory representation in primate prefrontal cortex: Evidence for a mnemonic hemianopia. Soc. Neurosci. Abstr. 12: 554.

Godschalk, M., R. N. Lemon, H. G. J. M. Kuypers, and H. K. Ronday (1984) Cortical afferents and efferents of monkey postarcuate area: An anatomical and electrophysiological study. Exp. Brain Res. 56: $410-424$.

Goldman, P.S. (1979) Contralateral projections to the dorsal thalamus 
from frontal association cortex in the rhesus monkey. Brain Res. 166: 166-171.

Goldman, P. S., and W. J. H. Nauta (1976) Autoradiographic demonstration of a projection from prefrontal association cortex to the superior colliculus in the rhesus monkey. Brain Res. 116: 145-149.

Goldman, P. S., and W. J. H. Nauta (1977a) Columnar distribution of cortico-cortical fibers in the frontal association, limbic and motor cortex of the developing rhesus monkey. Brain Res. 122: 393-413.

Goldman, P. S., and W. J. H. Nauta (1977b) An intricately patterned prefronto-caudate projection in the rhesus monkey. J. Comp. Neurol. 171: 369-386.

Goldman, P. S., and H. E. Rosvold (1970) Localization of function within the prefrontal cortex of the rhesus monkey. Exp. Neurol. 27: 291-304.

Goldman, P. S., H. E. Rosvold, B. Vest, and T. W. Galkin (1971) Analysis of the delayed alternation deficit produced by dorsolateral prefrontal lesions in the rhesus monkey. J. Comp. Physiol. Psychol. 77: 262-280.

Goldman-Rakic, P.S. (1987) Circuitry of the primate prefrontal cortex and the regulation of behavior by representational memory. In The Nervous System, Higher Functions of the Brain, Vol. 5, Handbook of Physiology, V. Mountcastle and F. Plum, eds., pp. 373-417, American Physiological Society, Washington, D.C.

Goldman-Rakic, P. S. (1988) Topography of cognition: parallel distributed networks in primate association cortex. Annu. Rev. Neurosci. 11: $137-156$.

Goldman-Rakic, P. S., and M. L. Schwartz (1982) Interdigitation of contralateral and ipsilateral columnar projections to frontal association cortex in primates. Scicnce 216: 755-757.

Goldman-Rakic, P. S., L. D. Selemon, and M. L. Schwartz (1984) Dual pathways connecting the dorsolateral prefrontal cortex with the hippocampal formation and parahippocampal cortex in the rhesus monkey. Neuroscience 12: 719-743.

Griffin, G., L. R. Watkins, and D. J. Mayer (1979) HRP pellets and slow-release gels: Two new techniques for greater localization and sensitivity. Brain Res. 168: 595-601.

Groves, P. M. (1983) A theory of the functional organization of the neostriatum and the neostriatal control of voluntary movement. Brain Res. Rev. 5: 109-132.

Hécaen, H., W. Penfield, C. Bertrand, and R. Malmo (1956) The syndrome of apractognosia due to lesions of the minor cerebral hemisphere. Arch. Neurol. Psychiatry 75: 400-434.

Hcilman, K. M., and E. Valenstein (1972) Frontal lobe neglect in man. Neurology 22: 660-664.

Holmes, G. (1918) Disturbances of visual orientation. Br. J. Opthalmol. 2: 449-468, 506-516.

Huerta, M. F., L. A. Krubitzer, and J. H. Kaas (1987) Frontal eye field as defined by intracortical microstimulation in squirrel monkeys. II. Cortical connections. J. Comp. Neurol. 265: 332-361.

Hyvärinen, J., and A. Poranen (1974) Function of the parietal associative area 7 as revealed from cellular discharges in alert monkeys. Brain 97: 673-692.

Jacobsen, S., N. Butters, and N. J. Tovsky (1978) Afferent and efferent subcortical projections of behaviorally defined sectors of prefrontal granular cortex. Brain Res. 159: 279-296.

Jones, E. G., and H. Burton (1976) Areal differences in laminar distribution of thalamic afferents in cortical fields of insular, parietal and temporal regions of primates. J. Comp. Neurol. 168: 197-248.

Jones, E. G., and T. P. S. Powell (1970) An anatomical study of converging sensory pathways within the cerebral cortex of the monkey. Brain 93: 793-820.

Jürgens, U. (1984) The efferent and afferent connections of the supplementary motor area. Brain Res. 300: 63-81.

Künzle, H. (1978) An autoradiographic analysis of the efferent connections from premotor and adjacent prefrontal regions (areas 6 and 9) in Macaca fascicularis. Brain Behav. Evol. 15: 185-234.

Kuypers, H. G. J. M., and D. G. Lawrence (1967) Cortical projections to the red nucleus and the brain stem in the rhesus monkey. Brain Res. 4: 151-188.

Leichnetz, G. R. (1980) An intrahemispheric columnar projection between two cortical multisensory convergence areas (inferior parietal lobule and prefrontal cortex): An anterograde study in macaque using HRP gel. Neurosci. Lett. 18: 119-124.

Leichnetz, G. R., R. F. Spencer, S. G. P. Hardy, and J. Astruc (1981) The prefrontal corticotectal projection in the monkey: An anterograde and retrograde horscradish peroxidase study. Neuroscience 6: 10231041 .

Leichnetz, G. R., D. J. Smith, and R. F. Spencer (1984) Cortical projections to the paramedian tegmental and basilar pons in the monkey. J. Comp. Neurol. 228: 388-408.

Lynch, J. C., A. M. Graybiel, and L. J. Lobeck (1985) The differential projection of two cytoarchitectonic subregions of the inferior parietal lobule in macaque upon the deep layers of the superior colliculus. J. Comp. Neurol. 235: 241-254.

Matelli, M., R. Camarda, M. Glickstein, and G. Rizzolatti (1986) Afferent and efferent projections of the inferior area 6 in the macaque monkey. J. Comp. Neurol. 251: 281-298.

Mesulam, M.-M. (1978) Tetramethylbenzidine for horseradish peroxidase neurochemistry: A non-carcinogenic blue reaction product with superior sensitivity for visualizing neural afferents and efferents. J. Histochem. Cytochem. 26: 106-117.

Mesulam, M.-M. (1981) A cortical network for directed attention and unilateral neglect. Ann. Neurol. 10:309-325.

Mountcastle, V. B., J. C. Lynch, A. Georgopoulos, H. Sakata, and C. Acuna (1975) Posterior parietal association cortex of the monkey: Command functions for operations within extrapersonal space. J. Neurophysiol. 38: 871-908.

Mountcastle, V. B., B. C. Motter, M. A. Steinmetz, and C. J. Duffy (1984) Looking and seeing: The visual functions of the parietal lobe. In Dynamic Aspects of Neocortical Function, G. M. Edelman, W. E. Gall, and W. M. Cowan, eds., pp. 159-193, Wiley, New York.

Mufson, E. J., and M.-M. Mesulam (1982) Insula of the Old World monkey. II. Afferent cortical input and comments on the claustrum. J. Comp. Neurol. 212: 23-37.

Pandya, D. N., and H. G. J. M. Kuypers (1969) Cortico-cortical connections in the rhesus monkey. Brain Res. 13: 13-36.

Pandya, D. N., and B. Seltzer (1982) Intrinsic connections and architectonics of posterior parietal cortex in the rhesus monkey. J. Comp. Neurol. 204: 196-210.

Pandya, D. N., and E. H. Yeterian (1984) Proposed neural circuitry for spatial memory in the primate brain. Neuropsychology 22: 109122.

Pandya, D. N., P. Dye, and N. Butters (1971) Efferent cortico-cortical projections of the prefrontal cortex in the rhesus monkey. Brain Res. $31: 35-46$.

Pearson, R. C. A., P. Brodal, K. C. Gatter, and T. P. S. Powell (1982) The organization of the connections between the cortex and the claustrum in the monkey. Brain Res. 234: 435-441.

Petrides, M., and D. N. Pandya (1984) Projections to the frontal cortex from the posterior parietal region in the rhesus monkey. J. Comp. Neurol. 228: 105-116.

Preuss, T. M., and P. S. Goldman-Rakic (1985) Somatosensory representation in primate prefrontal cortex: Connections of the principal sulcus with S-I, S-II, and adjacent areas of the frontoparietal operculum. Soc. Neurosci. Abstr. 11: 677.

Preuss, T. M., and P. S. Goldman-Rakic (1987) Crossed corticothalamic and thalamocortical connections of macaque prefrontal cortex. J. Comp. Neurol. 257: 269-281.

Rockland, K. S., and D. N. Pandya (1979) Laminar origins and terminations of cortical connections of the occipital lobe in the rhesus monkey. Brain Res. 179: 3-20.

Schwartz, M. L., and P. S. Goldman-Rakic (1982) Single cortical neurones have axon collaterals to ipsilateral and contralateral cortex in fetal and adult primates. Nature 299: 154-156.

Schwartz, M. L., and P. S. Goldman-Rakic (1984) Callosal and intrahemispheric connectivity of the prefrontal association cortex in rhesus monkey: Relation between intraparietal and principal sulcal cortex. J. Comp. Neurol. 226: 403-420.

Selemon, L. D., and P. S. Goldman-Rakic (1985) Longitudinal topography and interdigitation of corticostriatal projections in the rhesus monkey. J. Neurosci. 5: 776-794.

Seltzer, B., and D. N. Pandya (1978) Afferent cortical connections and architectonics of the superior temporal sulcus and surrounding cortex in the rhesus monkey. Brain Res. 149: 1-24.

Seltzer, B., and D. N. Pandya (1984) Further observations of parietotemporal connections in the rhesus monkey. Exp. Brain Res. 55:301312 .

Seltzer, B., and G. W. Van Hoesen (1979) A direct inferior parietal lobule projection to the presubiculum in the rhesus monkcy. Brain Res. 179: 157-162. 
van Essen, D. C., and J. H. R. Maunsell (1983) Hierarchical organization and functional streams in the visual cortex. TINS 6:370-375.

Vogt, C., and O. Vogt (1919) Allgemimere ergebnisse unserer hirnforschung. J. Psychol. Neurol. 25: 279-461.

von Bonin, G., and P. Bailey (1947) The Neocortex of Macaca mulatta, University of Illinois Press, Urbana, IL.
Yeterian, E. H., and D. N. Pandya (1985) Corticothalamic connections of the posterior parietal cortex in the rhesus monkey. J. Comp. Neurol. 237: 408-426.

Yeterian, E. H., and G. W. Van Hoesen (1978) Cortico-striate projections in the rhesus monkey: The organization of certain corticocaudate connections. Brain Res. 139: 43-63. 